\title{
Fuzzy Based Multi-Fever Symptom Classifier Diagnosis Model
}

\author{
Ighoyota Ben Ajenaghughrure and Dr. P. Sujatha \\ Vels University, Department of computer science, Chennai, 600117, India \\ Ighoyotaben@yahoo.com, suja.research@gmail.com \\ Dr. Maureen I. Akazue \\ Delta State University, Department of Computer Science, Abraka, 330106, Nigeria \\ Akazuem@gmail.com
}

Received: 08 November 2016; Accepted: 31 August 2017; Published: 08 October 2017

\begin{abstract}
Fever has different causes and types, but with similar symptoms. Therefore, making fever diagnosis with human physiological symptoms more complicated. This research project delves into the design of a web based expert multi-fever diagnosis system using a novel fuzzy symptom classifier with human self-observed physiological symptoms. Considering malaria, Lassa, dengue, typhoid and yellow fever. The fuzzy-symptom classifier has two stages. Fist stage is fever type confirmation using common fever symptoms, leading to five major fuzzy rules and the second phase is determining the level of infection (severe or mild) of the confirmed type of fever using unique fever symptoms. Furthermore, Case studies during the system implementation yielded data collected from 50 patients of having different types of fever. The analysis clearly shows the effectiveness and accuracy in the system performance through false result elimination. In addition, acceptability of the system was investigated through structured questionnaire administered to same 50 patients. This result clearly indicates that the system is well accepted, by users and considered fairly easy to use, time and cost saving.
\end{abstract}

Index Terms-Fuzzy classifier, fever diagnosis, multi fever, expert fever diagnosis.

\section{INTRODUCTION}

Fever is a change in the human body temperature, both minimum and maximum [4],[5]. During this condition, a sufferer generally experiences cold and muscle seizure [3], resulting to alteration of body temperature regulation system producing more heat in a bid to sustain normal body temperature, which when restored, leads to excessive sweating[25]. Although there is discrepancy in the normal human temperature [1],[2], but fever occurs in body temperature between the range 41 to $42{ }^{\circ} \mathrm{C}(105.8$ to $\left.107.6^{\circ} \mathrm{F}\right)[5]$.

The causes of fever varies from infectious to noninfectious diseases, but the case of body temperature changes also referred to as hypothermia is completely different[7]. Since it is not a result of either causative above. Treatment of both fever and hyperthermia to reduce or subside its presence is not necessary[6],[24], but a direct treatment of its associated symptoms such as muscle pains, headache etc is considered more useful[8], using common drug such as paracetamol to more intensive care methodology, depending on the severity of the sufferers health status[8],[9]. Being a common symptom of most health problem, fever is accountable for approximately $30 \%$ of children healthcare-centers visit [6] and dominates up to $75 \%$ of critically ill adults [10].

The common types of fever widely reported with scientifically available medication includes malaria, dengue, typhoid, Lassa, and yellow fever. These has great similarities in symptom's irrespective of carriers, infection type(bacteria, virus etc) and treatment. This relationship between various types of fever symptoms, makes diagnosis of fever with human physiological symptoms is difficult, for example symptoms of Lassa is very hard to differentiate from those of malaria, yellow, dengue and typhoid fever [11],[12]. In addition to the high cost associated with acquiring wet lab fever diagnosis service, lack of medical expert availability and accessibility, has prompted the development of computer aided expert fever diagnosis systems for diagnosing single or multiple types of fever. Unfortunately, the existing computer based expert fever diagnosis systems do not take symptoms relationship into consideration, which has significant impact on the accuracy of the fever diagnosis results they produce. To overcome this challenge, a fuzzy-based multi-fever symptom classifier for fever diagnosis implemented as a web system for accessibility is proposed and designed in this research. The fuzzy-based multi-fever symptom classifier put into consideration all the various types of fever related symptoms, and accurately determine the type fever and level of infection (mild/acute) a patient is suffering from, based on user input of physiological symptoms. The implemented fuzzy-based multi-fever symptoms classifier is known as e-fever portal. Fuzzy technique derived from artificial intelligence was specifically chosen, because of its longstanding successful application 
in health related application. This research only considers the use of human physiological symptoms to diagnose fever. In addition, it does not take into account a critically ill patient who cannot speak, walk e.tc. Furthermore, it does not serve as a total replacement for expert medical practitioners, but as an assistive solution to fever diagnosis. Finally our study solely encompasses five types of fever (malaria, dengue, yellow, Lassa and typhoid).

This research paper is divided into seven sections. The first section is an introductory overview of the research paper, while the second is review of related literature. The third section is a comparative study between existing fever diagnosis system and the proposed fuzzy based multi-fever symptom classifier diagnosis system. Furthermore, the fourth section encompasses the design of the fuzzy based multi-fever symptom classifier and the e-fever web application architecture. The fifth section is the implementation and acceptability data analysis of the proposed system. The sixth section is conclusion based on findings from the system implementation.

\section{RELATED WORKS}

The application of artificial intelligence techniques in fever diagnosis towards optimizing diagnostics results accuracy has been adopted by several researcher amongst which includes: (1) [26], which developed a system named diagnosis portal for human disease diagnosis based on human physiological symptom. The system did not focus on fever diagnosis, but exhibited the applicability and importance of fuzzy logic in medical diagnosis. Disease such as sleep apnea, irritable bowel syndrome, and attention deficit hyperactivity disorder. This project further proved and support the long growing research in application of fuzzy in medical diagnosis. (2) In addition, [27] carried out an in-depth review on the applications of fuzzy logic in expert medical diagnosis systems, as an important technique useful in expert medical system. Through in-depth review on existing fuzzy expert medical diagnosis system. Their result shows significant success and adaption of artificial intelligence technique in expert computer aided medical diagnosis systems. (3) Furthermore, to enhance the accuracy of artificial intelligent based expert medical diagnosis system, [28] developed a genetic algorithm to enhance optimum performance of neuro-fuzzy systems used for diagnosis of typhoid. This is to ensure accuracy of result, which in an ordinary neuron fuzzy based typhoid fever diagnosis system, is computed with errors. This is an advancement on the performance of typhoid fever diagnosis systems based on neuro-fuzzy, but its applicability on other fever types was not considered, as well as symptoms interrelationship among different fever types was not also considered in the neurofuzzy system. (4) A computer aided malaria fever diagnosis system was developed using the rough set theory machine learning technique, implemented as a web based application [20]. While the correctness of the result of the training and testing data are $100 \%$ and $94 \%$ being promising solution.
It lack the consideration of various fever symptoms relationships which could hinder the correctness of malaria fever diagnosis in the case of multiple infection [20]. (5) Furthermore, [21] developed a web based multifever diagnosis system based on simple if-then rules for diagnosing malaria, dengue, typhoid, Lassa, Hay, peeebstein, leptospirosisscarlet and rheumatic fever. Quiet an integrated novel system, but the result will be prone to error due to the lack of the various types of fever symptoms relationships consideration. Although it promises to speedup medical diagnosis and treatment administration time. (6) Also, [22] proposed a fuzzy logic based malaria fever diagnosis system, using UML simulation, with the sole aim of speeding up the diagnosis process, time and reducing embodiment on medical professionals. Although quiet promising due to the optimistic success shown by fuzzy application in medical diagnosis this system lacks implementation. (7) In addition, [23] developed a malaria fever diagnosis system incorporating intelligence and expertise using fuzzy logic. The results of these systems reviewed so far seem accurate and perfect solution for timely and effective diagnosis of malaria, if only malaria fever symptoms are reported by patients and are not similarly to other types of fever general symptoms, which is not possible. Hence rendering the results from existing system in question of accuracy since malaria fever share some common symptoms with other types of fever.

Most Computer aided expert fever diagnosis systems developed by researchers so far are broadly classified into two categories as either multi or single fever diagnosis system. S. Govinda et al [29] developed a ruled based expert multi fever diagnosis system that diagnoses dengue, malaria and typhoid fever using fuzzy logic. The result is the type of fever, its level of infection (severe or mild), and recommended food and drugs. This system fail to consider the interlink between types of fever through symptoms similarity, hence the diagnosis results accuracy cannot be ascertain in this context. Furthermore, [19] developed a fuzzy based malaria and dengue fever diagnosis system. The system was developed using MATLAB fuzzy toolbox GUI. This novel system, diagnoses malaria and dengue fever effectively, but lack consideration of interrelationship of symptoms, making the result of this system uncertain in terms of validity.

While single fever diagnosis systems, as seen in [14], which developed a clinical machine learning based expert typhoid fever diagnosis system,. Although the result from this research seem promising, as a computer based typhoid fever diagnosis system with implementation and therapy, but it fails to put into account the symptoms relationship between typhoid and other fever types, which when considered renders the diagnosis rule developed here invalid as some will lead to other fever types. Also, [15] developed a non-invasive cost effective adaptive neuron fuzzy based dengue fever patient risk level diagnosis system, to diagnose dengue fever accurately and determine the risk level of a patient. In order to avoid unwanted hospitalization and its associated cost. Although the system seem effective, but solely 
concentrated on dengue fever and its symptoms alone, hence making its diagnosis results accuracy subject to wet lab test, due to its non-inclusion of system relationship. In addition, [16] developed a web-based expert system to diagnose dengue fever. This system enables easy access to diagnosis and self-diagnosis of dengue fever from normal fever. Quit novel, but its results lack the credibility, as the project did not consider symptom relationship, which has great impact on the diagnosis result. Furthermore, [17] developed a fuzzy logic based expert typhoid fever diagnosis system. The system is a web application to enable accessibility. Unfortunately, the system still did not consider symptoms relationship, which renders the validity of the system diagnosis result uncertain. In addition, [18] developed a fuzzy expert system for management of malaria. Although quit novel, the system did not also consider symptoms relationships among the various types of fever, hence, leaving the results from the system uncertain, in terms of validity and accuracy, despite the practical approach towards real life data collection in addition to the fact that it only considered malaria fever symptoms.

Hence, the proposed fuzzy-based multi-fever symptom classifier is a novel method that promises to optimize the accuracy of multi-fever diagnosis.

\section{COMPARATIVE ANALYSIS OF EXISTING AND PROPOSED EXPERT FEVER DIAGNOSIS SYSTEMS}

The table1 shows analysis comparing some existing expert fever diagnosis system, using indicators such as single or multiple fever diagnosis capability, inclusion of artificial intelligence technique, therapy recommendation, symptom relationship from numerous types of fever well successful in medical diagnosis application, symptom relationship and implementation. The numbers $29,14,15$, $16,17,18,19,20,21,22$, and 23 are references for articles containing the respective expert fever diagnosis systems under review for comparison.

We can clearly see that not all-existing system considered the impact of fevers symptoms relationship, as an important factor during fever diagnosis using human physiological symptom, which can affect the diagnosis result negatively.

Table 1. Comparative analysis of existing expert fever diagnosis systems and our proposed fever diagnosis system $29,14,15$, $16,17,18,19,20,21,22$, and 23

\begin{tabular}{|c|c|c|c|c|c|c|c|c|c|c|c|c|}
\hline Indicators & {$[29]$} & {$[14]$} & {$[15]$} & {$[16]$} & {$[17]$} & {$[18]$} & {$[19]$} & {$[20]$} & {$[21]$} & {$[22]$} & {$[23]$} & $\begin{array}{c}\text { proposed } \\
\text { system }\end{array}$ \\
\hline Multi fever & Yes & Yes & No & No & No & No & No & Yes & No & No & Yes & Yes \\
\hline Single fever & No & No & Yes & Yes & Yes & Yes & Yes & Yes & Yes & Yes & Yes & No \\
\hline $\begin{array}{c}\text { Artificial } \\
\text { intelligence } \\
\text { technique }\end{array}$ & Yes & Yes & Yes & Yes & No & Yes & Yes & Yes & Yes & Yes & Yes & Yes \\
\hline $\begin{array}{c}\text { Symptoms } \\
\text { Relationship }\end{array}$ & No & No & No & No & No & No & No & No & No & No & No & Yes \\
\hline Implementation & No & Yes & No & No & Yes & Yes & Yes & Yes & Yes & Yes & Yes & Yes \\
\hline
\end{tabular}

Furthermore, the authors of [29], [19] and [21] research work attempted diagnosing multiple fever in their system using artificial intelligence technique. While the authors of [15], [16], [17], [18], [19], [20], [21], [22] and [23] designed a single fever diagnosis system. To further support the importance of artificial intelligence technique in medical diagnosis, the systems developed by authors in [29], [14],[15], [16], [18], [19], [20], [21], [22], and [23] all utilized artificial intelligence technique. Furthermore, we can infer that only our proposed system has multi-fevers symptoms relationship considered, hence making it not just a novel system, but necessary for effective diagnosis of fever as a result of the complication involved in physiological symptoms based diagnosis of fever.

\section{IMULTI-FEVER SYMPTOM RELATIONSHIP}

An indepth study was conducted on malaria, dengue,
Lassa, yellow and typhoid fever symptoms to identify common symptoms. During which, literature review and interview were used as the technique for collecting information about these types of fever. The results reveal symptoms relationship between malaria, dengue Lassa, yellow and typhoid fever. These common symptoms were classified as general symptoms, while symptoms unique to each type of fever were classified as unique. It can be inferred that fever is the most common symptom of all the five types of fever under study. Followed by headache and vomiting, that is common to four. Next common symptoms are conjunctiva, nausea, abdominal pain, diarrhea, malaise, and muscular pains, which are common to only three types of fever randomly permuted. Finally severe hepatitis, shivering/chills, cough, and mucosal bleeding are the less common symptoms, but found in any two type of fever under study. All other symptoms are unique to a particular fever, or common to two or three types respectively. 
Table 2. Fever symptom relationship

\begin{tabular}{|c|c|c|c|c|c|c|}
\hline $\mathrm{S} / \mathrm{N}$ & Symptom & $\begin{array}{l}\text { Yellow } \\
\text { fever }\end{array}$ & $\begin{array}{l}\text { Typhoid } \\
\text { fever }\end{array}$ & $\begin{array}{c}\text { Malaria } \\
\text { fever }\end{array}$ & $\begin{array}{l}\text { Lassa } \\
\text { fever }\end{array}$ & $\begin{array}{c}\text { Dengue } \\
\text { fever }\end{array}$ \\
\hline 1. & Fever & yes & yes & Yes & yes & Yes \\
\hline 2. & Headache & yes & yes & Yes & no & Yes \\
\hline 3. & Myalgia & yes & no & No & no & \\
\hline 4. & Conjunctiva infection & yes & no & No & yes & No \\
\hline 5. & Facial flushing & yes & no & No & no & No \\
\hline 6. & lumbosacral pain & yes & no & No & no & No \\
\hline 7. & Nausea & yes & no & Yes & yes & No \\
\hline 8. & Vomiting & yes & no & Yes & yes & Yes \\
\hline 9. & abdominal pain & yes & no & No & yes & Yes \\
\hline 10. & $\begin{array}{l}\text { Impaired level of } \\
\text { consciousness }\end{array}$ & yes & no & No & no & No \\
\hline 11. & Severe hepatitis & yes & yes & No & no & No \\
\hline 12. & Shock & Yes & No & No & No & No \\
\hline 13. & Constipation & no & yes & No & no & No \\
\hline 14. & Diarrhea & no & yes & Yes & yes & No \\
\hline 15. & Jaundice & yes & no & No & no & No \\
\hline 16. & Malaise & no & yes & Yes & yes & No \\
\hline 17. & Sore throat & no & No & No & yes & No \\
\hline 18. & Shivering / chills & no & No & Yes & yes & No \\
\hline 19. & Cough & no & yes & No & yes & No \\
\hline 20. & Muscular pains & no & No & Yes & yes & Yes \\
\hline 21. & chest pain & no & No & No & yes & No \\
\hline 22. & Back pain & no & No & No & yes & No \\
\hline 23. & Sweating & no & No & Yes & no & No \\
\hline 24. & Fatigue & no & No & Yes & no & No \\
\hline 25. & Facial Swelling & no & No & No & yes & No \\
\hline 26. & Mucosal bleeding & no & No & No & yes & Yes \\
\hline 27. & Myocarditis & no & Yes & No & no & No \\
\hline 28. & Pneumonia & no & Yes & No & no & No \\
\hline 29. & Anorexia. & no & Yes & No & no & No \\
\hline 30. & Exanthema (rose spots), & no & Yes & No & no & No \\
\hline 31. & Skin rash (measles-like) & no & No & No & no & Yes \\
\hline 32. & Lethargy or restlessness & no & No & No & no & Yes \\
\hline
\end{tabular}

\section{Design Of Fuzzy Based Multi-Fever Diagnosis SYMPTOM CLASSIFIER}

As depicted in table 2 above, its is obvious that there are similarities in symptoms among various types of fever, hence rendering fever diagnosis more difficult with physiological signs. Therefore, the fuzzy based multifever symptoms classifier for accurate diagnosis proposed in this study is designed in this section as shown in the fig1. The fuzzy based multi-fever symptom classifier consists of three major stages. At the first stage, each input symptom is classified as general symptom and unique symptom in order of entry. The general symptoms input are arranged as the first sets of input variables of the fuzzy model. These will determine the type of fever a patient is suffering from. While the unique symptoms are used to determine the level of infection of the confirmed type of fever. The second stage is a further classification of the symptoms as mild or severe, to determine the level of infection of the confirmed type of fever using membership function and rule. The third level is the inference engine of the fuzzy based symptom classifier that comprises several rules to determine the output of the fuzzy classifier. The finally stage is the output stage, which gives two results the type of fever confirmed and the level of infection. Hence, the fuzzy based classifier is a multi-input and multi-output fuzzy model. Where all the five types of fever symptoms serves as input to the fuzzy system, and two types of output corresponding to fever types confirmed and the level of infection of the confirmed type of fever(severe or mild) are produced. 


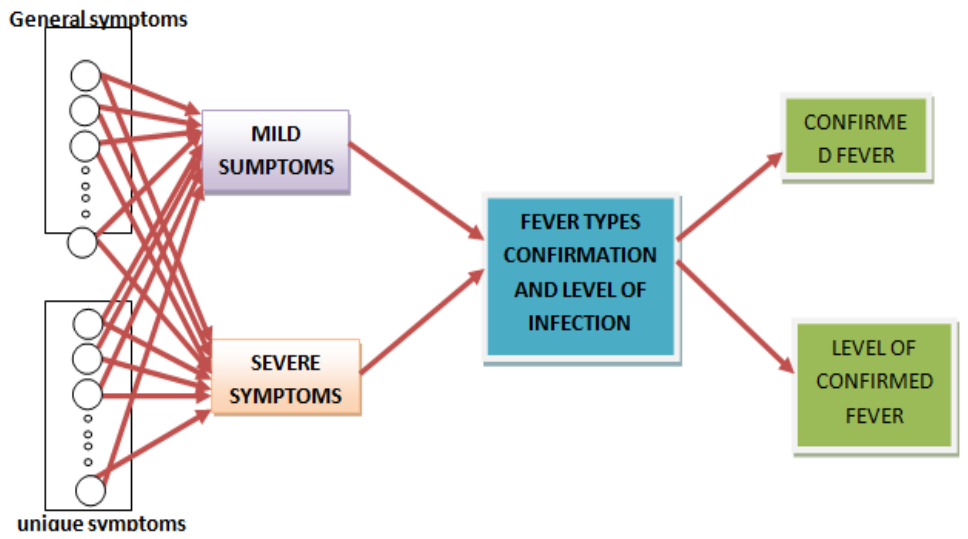

Fig.1. Proposed model of fuzzy based multi-fever ymptom classifier for e-fever portal

\section{A. Fuzzy system components}

\section{- Input:}

The fuzzy based multi-fever symptom classifier Model for the e-fever portal inputs variables are the symptoms responses from users. These responses are classified into the following fuzzy linguistics variables and their corresponding values as recorded table 3 .

Table 3. Fuzzy based multi-fever symptom classifier model for e-fever portal input variables linguistics and numerical values

\begin{tabular}{|c|c|c|}
\hline S/N & Input variables & Fuzzy values \\
\hline 1 & $\begin{array}{c}\text { Mild symptom(this can be general } \\
\text { or unique symptom) }\end{array}$ & 0.55 \\
\hline 2 & $\begin{array}{c}\text { Severe symptom(this can be } \\
\text { general or unique symptom) }\end{array}$ & 1.0 \\
\hline 3 & Mild infection & $1.1<=4.4$ \\
\hline 4 & Severe infection & $4.65<=12.75$ \\
\hline
\end{tabular}

These values for the linguistics variable are mapped to all the symptoms in table2. The general and unique symptom classification is silent but very important to the diagnosis process through the arrangement of the system input variable; therefore, all first few inputs are general symptoms, while the last sets of inputs are unique symptoms. Similarly, in the e-fever portal implementation of the fuzzy classifier, patients respond to general symptoms questions before unique symptoms related question.

The triangular membership (MF) is used which Is specified by the three parameters $\{a, b, c\}$ for each membership function $\mathrm{x}=$ (mild, severe), denoted mathematically as

Fever symptom $(\mathrm{A}: \mathrm{a}, \mathrm{b}, \mathrm{c})=\max \left(\min \left(\frac{x-a}{b-1}, \frac{c-x}{c-b}\right), 0\right)[13]$

Where parameters $\mathrm{a}, \mathrm{b}, \mathrm{c}$ determines the $\mathrm{x}$ coordinates of the three corners of the underlying triangular membership function.

\section{- Fuzzy inference engine/system:}

This is the brain and Intelligence of the fuzzy based multi-fever symptom classifier for the e-fever portal. it takes the premise as input and produces different consequences as output. The premise here are the symptoms, which are represented with numerical values and the consequence here, is the type of fever confirmed and the level of infection. Hence, the inference engine is referred to as the fuzzy inference system, mainly consisting of the fuzzy RULE, which is described in detail below

Rules: the fuzzy system rules, first confirms the type of fever, and then confirm the level of infection consequently. Following table 2 analysis, we can clearly infer that Fever, Headache, Vomiting, Nausea, Abdominal pains are the most common symptoms among the five types of fever under study. Hence, our fuzzy rule uses these five symptoms to confirm the type of fever infection, before further investigation into the level of infection of the confirmed type of fever is done. The first five rules confirms the various types of fever without level of infection known, next an in-depth analysis base on further user responses to symptoms, reveals the level of infection.

- If fever =yes and headache=yes and vomiting=yes, and nausea=yes and abdominal pain=yes then fever type $=$ yellow fever and level of infection $=$ unknown

- If fever =yes and headache=yes and vomiting=yes, and nausea $=y e s$ and abdominal pain $=$ no and others $=$ no then fever type $=$ malaria fever and level of infection = unknown

- If fever =yes and headache=yes and vomiting=yes, and nausea $=$ no and abdominal pain=yes and others $=$ no then fever type $=$ dengue fever and level of infection $=$ unknown

- If fever =yes and headache=yes and vomiting=no, and nausea $=$ no and abdominal pain $=$ no and others $=$ no then fever type $=$ typhoid fever and level of infection $=$ unknown 
- If fever =yes and headache=no and vomiting=yes, and nausea=yes and abdominal pain=no others = no then fever type =Lassa fever and level of infection $=$ unknown

\section{B. Fever Type Confirmation And Infection Level} Detection Technique:

Symptoms already hold numerical values classifying them as either mild or severe symptoms, as seen in table 3 for all symptom in table2. Hence determining the type of infection, and the level of infection, can be done by summing up all the symptoms numerical values, for each patient response during diagnosis. Mathematically expressed below:

$$
\begin{gathered}
\mathrm{F}_{\mathrm{x}}=\mathrm{M}_{\text {symptom }}+\mathrm{S}_{\text {symptom }} \\
\mathrm{M}_{\text {symptom }}=\sum_{1}^{n} S V \\
\mathrm{~S}_{\text {symptom }}=\sum_{1}^{n} S V
\end{gathered}
$$

Where SV is symptom corresponding numeric value for each class of symptom.

$\mathbf{M}_{\text {symptom }}$ is the sum of all the numerical values corresponding to general symptoms for confirming the type of fever that are responded to by patient during diagnosis.

$\mathrm{S}_{\text {symptom }}$ is the sum of all the numerical values corresponding to unique symptoms for confirming the type of fever that are responded to by patient during diagnosis.

$$
\begin{gathered}
Y=\text { all symptoms values (i). } \\
i=\text { number of symptoms. }
\end{gathered}
$$

Where $\mathrm{x}=$ any fever (malaria, dengue, Lassa, yellow, typhoid) confirmed and the level of infection

\section{Expert System Design:}

The proposed e-fever portal implementing the novel fuzzy classifier designed comprises of two modules. The first module is an educational module, consisting of additional five-sub module. Each education modules contains information relevant to each of the five types of fever under study, their causes, symptoms and few precautionary measures. While the second module is the diagnosis module implementing the fuzzy based classifier designed in this research project as a two state system, corresponding to two more sub module, the first diagnosis sub module confirms the type of fever, using the general symptoms. In addition, the last diagnosis module determines the level of infection of the confirmed type of fever

\section{- Education module:}

This module consists of detailed information about the various types of fever. it is subdivided into five modules, each of which consist of detailed information about the various types of fever (lassa, dengue, malaria, typhoid and yellow fever) . Such as, symptoms, history, geographical location infected, treatments and general advices

\section{- Diagnosis Module:}

This is the module where patient self-diagnose themselves to identify the type of fever they are suffering from, through inputted self

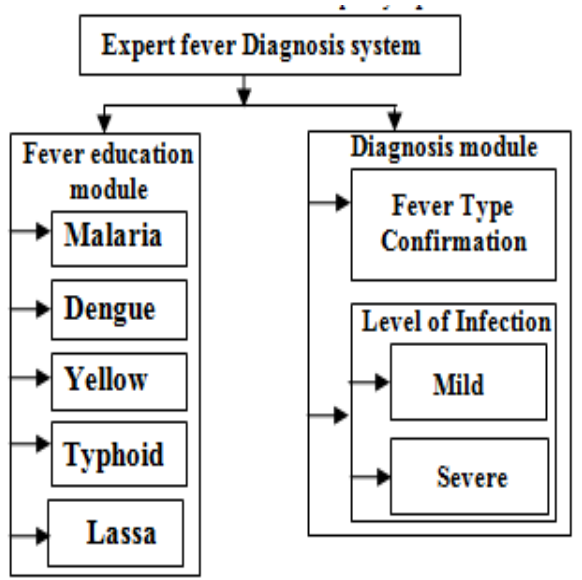

Fig.2. E-fever portal architecture

Diagnosis flow chart of the e-fever portal: fig 3 depicts the typical flowchart of the e-fever portal. It consists of two stages. Firstly begins with fever symptom, if a response this question is "NO", then there is no point proceeding further, as the user is currently not suffering from fever. Else, it continues to ascertain the type of fever the patient is suffering from, before advancing to the second stage to identify the level of infection using unique symptoms of the confirmed type of fever

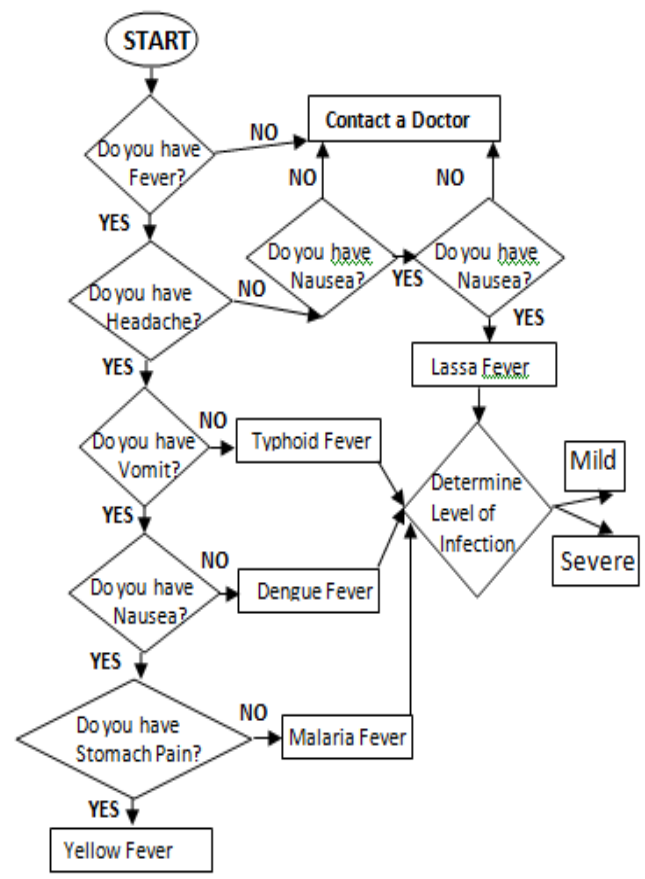

Fig.3. Fuzzy based integrated expert fever diagnosis system Flowchart 


\section{DATA ANALYSIS}

Data were collected from several fever patients with medial records cards from government and privately operated health centers. A total of 50 participants were exposed to the e-fever portal. $28 \%$ were female and $72 \%$ male, with an average age of 25years. Their response to the symptoms questions were recorded automatically into the system database. As presented in table4. The collected data were analyzed using descriptive statistic technique (bar char, pie chart histogram etc) for the sole purpose of clarity and better interpretation of the results.

Table 4. Sample data of various fever patients

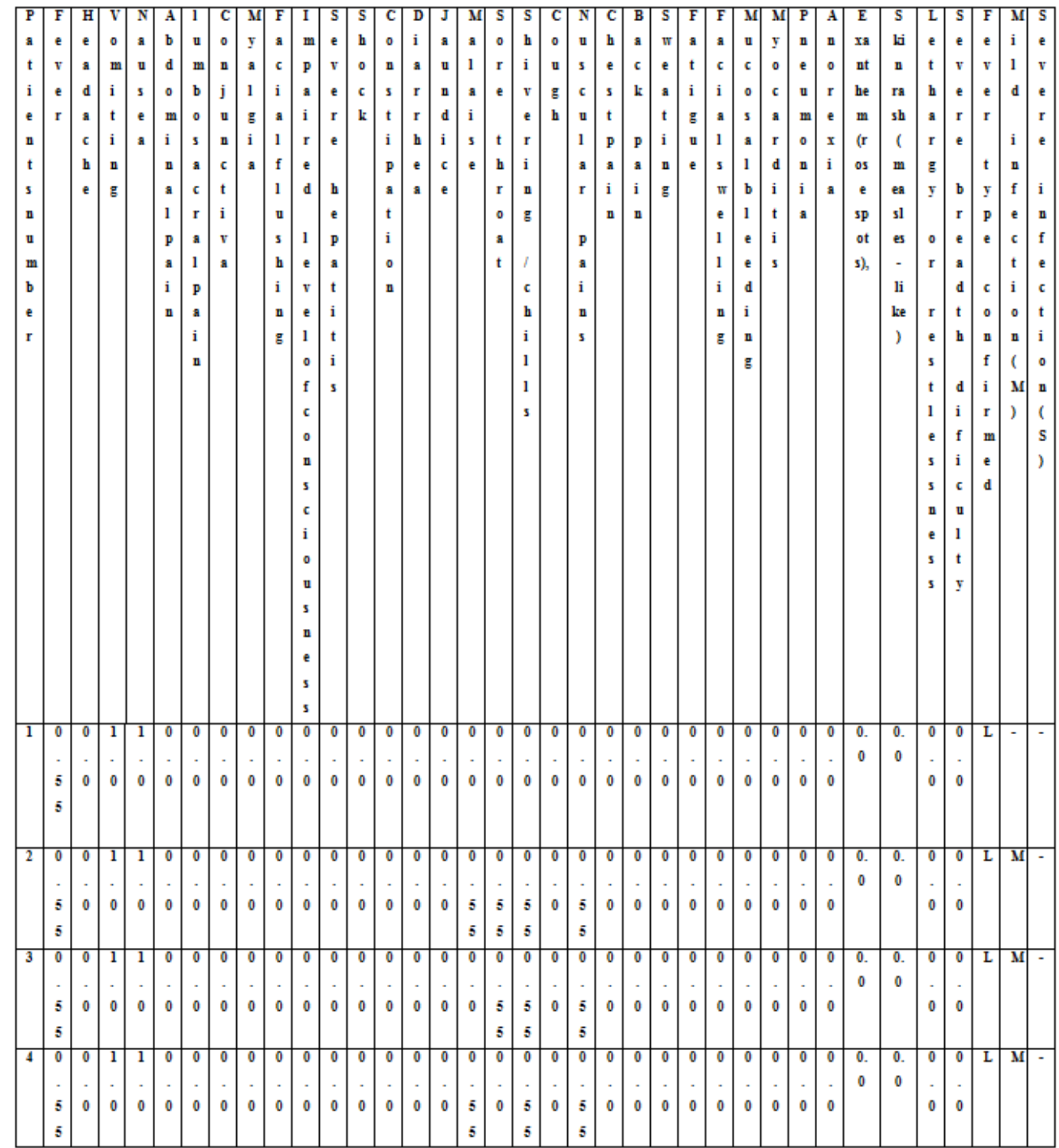




\begin{tabular}{|c|c|c|c|c|c|c|c|c|c|c|c|c|c|c|c|c|c|c|c|c|c|c|c|c|c|c|c|c|c|c|c|c|c|c|}
\hline \multicolumn{2}{|r|}{$\begin{array}{l}0 \\
5 \\
5 \\
5\end{array}$} & \begin{tabular}{l|l}
0 \\
0 \\
0
\end{tabular} & \begin{tabular}{l|l} 
& \\
- & \\
0 &
\end{tabular} & \begin{tabular}{|l|}
1 \\
- \\
0
\end{tabular} & . & i & 0 & 0 & 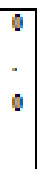 & \begin{tabular}{l|l} 
\\
- \\
0
\end{tabular} & $\begin{array}{l} \\
0 \\
0\end{array}$ & \begin{tabular}{l|l}
0 \\
- \\
0
\end{tabular} & . & 0 & \begin{tabular}{l|l}
0 \\
- \\
0
\end{tabular} & \begin{tabular}{l|l}
0 \\
0 \\
0
\end{tabular} & \begin{tabular}{l|l} 
& \\
5 & \\
5 & \\
5 &
\end{tabular} & & $\begin{array}{ll}. & 0 \\
0 & 0\end{array}$ & \begin{tabular}{l|l} 
& 0 \\
. & \\
0 & 0
\end{tabular} & \begin{tabular}{l|l} 
& 0 \\
. & \\
0 & 0 \\
0 & 0
\end{tabular} & \begin{tabular}{l|l}
0 & 0 \\
0 & 0 \\
0
\end{tabular} & | & \begin{tabular}{l|} 
\\
\\
0
\end{tabular} & \begin{tabular}{|l|}
0 \\
0 \\
0
\end{tabular} & \begin{tabular}{l|l} 
\\
0 \\
0
\end{tabular} & 0 & \begin{tabular}{l|l}
0 \\
0 \\
0
\end{tabular} & \begin{tabular}{l|} 
\\
\\
0
\end{tabular} & & & 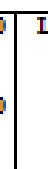 & \begin{tabular}{|l|l|}
$\mathrm{M}$ & $\mathbf{M}$
\end{tabular} & 1 \\
\hline$\overline{6}$ & $\begin{array}{l}0 \\
5 \\
5 \\
5\end{array}$ & $\begin{array}{l}0 \\
0 \\
0\end{array}$ & $\begin{array}{l}1 \\
. \\
0\end{array}$ & $\begin{array}{l}1 \\
- \\
0\end{array}$ & 0 & 0 & 0 & 0 & 0 & $\begin{array}{l}0 \\
. \\
0\end{array}$ & $\begin{array}{l}0 \\
- \\
0\end{array}$ & $\begin{array}{l} \\
0 \\
0\end{array}$ & $\begin{array}{l}0 \\
0 \\
0\end{array}$ & $\begin{array}{l}0 \\
- \\
0\end{array}$ & $\begin{array}{l}0 \\
- \\
0\end{array}$ & $\begin{array}{l} \\
\\
0\end{array}$ &. & \begin{tabular}{l|l}
0 & \\
. & \\
5 & \\
5 &
\end{tabular} & \begin{tabular}{l|l} 
& 0 \\
0 &. \\
0 & 0 \\
0 & 0
\end{tabular} & $\begin{array}{l}0 \\
0 \\
0\end{array}$ & . & $\begin{array}{l}0 \\
0 \\
0\end{array}$ & $\begin{array}{l}0 \\
0\end{array}$ & \begin{tabular}{|l|} 
\\
- \\
0
\end{tabular} & $\begin{array}{l}0 \\
0 \\
0\end{array}$ & \begin{tabular}{l|l} 
\\
0 \\
0
\end{tabular} & 0 & \begin{tabular}{l|} 
\\
0 \\
0 \\
0
\end{tabular} & $\begin{array}{l} \\
0 \\
0\end{array}$ & & 0 & 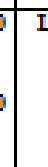 & $\mathbf{M}$ & \\
\hline 7 & 5 & $\begin{array}{l}0 \\
0 \\
0\end{array}$ & $\begin{array}{l}1 \\
0 \\
0\end{array}$ & $\begin{array}{l}1 \\
0 \\
0\end{array}$ & 0 & $i_{0}$ & 1 & 0 & : & \begin{tabular}{l|l} 
\\
0 \\
0
\end{tabular} & $\begin{array}{l}0 \\
- \\
0\end{array}$ & $\begin{array}{l}0 \\
0 \\
0\end{array}$ & $\begin{array}{l}0 \\
0 \\
0\end{array}$ & $\begin{array}{l}1 \\
- \\
0\end{array}$ & $\begin{array}{l} \\
0 \\
0\end{array}$ & $\begin{array}{l}0 \\
5 \\
5 \\
5\end{array}$ & \begin{tabular}{l|l} 
& \\
5 & \\
5 & \\
5 &
\end{tabular} & \begin{tabular}{l|l}
0 & \\
5 &. \\
5 &
\end{tabular} & \begin{tabular}{l|l}
1 & 0 \\
. & \\
0 & 5 \\
0 & 5
\end{tabular} & $\begin{array}{l}0 \\
5 \\
5 \\
5\end{array}$ & \begin{tabular}{l|}
1 \\
0 \\
0
\end{tabular} & \begin{tabular}{l|l}
1 & 0 \\
& 0 \\
0 & 0
\end{tabular} & $\begin{array}{l}0 \\
0\end{array}$ & \begin{tabular}{|l|} 
\\
0 \\
0
\end{tabular} & $\begin{array}{l}1 \\
0 \\
0\end{array}$ & \begin{tabular}{l|l} 
\\
0 \\
0
\end{tabular} & 0 & $\begin{array}{l}0 \\
0 \\
0\end{array}$ & 0 & & 0 & 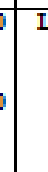 & - & \\
\hline 8 & $\begin{array}{l}- \\
5 \\
5\end{array}$ & $\begin{array}{l} \\
0 \\
0\end{array}$ & $\begin{array}{l}1 \\
- \\
0\end{array}$ & $\begin{array}{l}1 \\
0\end{array}$ & 0 & 0 & 0 & 0 & 0 & $\begin{array}{l} \\
0 \\
0\end{array}$ & $\begin{array}{l}0 \\
0\end{array}$ & $\begin{array}{l}0 \\
0 \\
0\end{array}$ & $\begin{array}{l}0 \\
. \\
0\end{array}$ & $\begin{array}{l}1 \\
0\end{array}$ & $\begin{array}{l}0 \\
0 \\
0\end{array}$ & $\begin{array}{l}0 \\
5 \\
5 \\
5\end{array}$ & $\begin{array}{l}0 \\
5 \\
5 \\
5\end{array}$ & $\begin{array}{l}0 \\
5 \\
5 \\
5\end{array}$ & \begin{tabular}{l|} 
\\
\\
0
\end{tabular} & $\begin{array}{l}5 \\
5 \\
5\end{array}$ &. & $\begin{array}{l}1 \\
0 \\
0\end{array}$ & j & \begin{tabular}{l|l}
1 \\
0 \\
0
\end{tabular} & $\begin{array}{l}1 \\
0 \\
0\end{array}$ & \begin{tabular}{l|l} 
\\
0 \\
0
\end{tabular} & 0 & $\begin{array}{l}0 \\
0 \\
0\end{array}$ & $\begin{array}{l}0 \\
.\end{array}$ & & 0 & 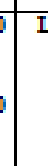 & - & \\
\hline 5 & \begin{tabular}{l|}
0 \\
5 \\
5 \\
5
\end{tabular} & $\begin{array}{l} \\
0 \\
0\end{array}$ & $\begin{array}{l}1 \\
- \\
0\end{array}$ & $\begin{array}{l}1 \\
0 \\
0\end{array}$ & j & 0 & 0 & 0 & 0 & \begin{tabular}{l|l}
0 \\
0 \\
0
\end{tabular} & $\begin{array}{l}0 \\
- \\
0\end{array}$ & $\begin{array}{l}0 \\
0 \\
0\end{array}$ & $\begin{array}{l}0 \\
0 \\
0\end{array}$ & $\begin{array}{l} \\
0 \\
0\end{array}$ & $\begin{array}{l}0 \\
- \\
0\end{array}$ & $\begin{array}{l}0 \\
0 \\
0\end{array}$ & \begin{tabular}{l|}
5 \\
5
\end{tabular} & $\begin{array}{l}5 \\
5\end{array}$ & $\begin{array}{l}0 \\
0 \\
0\end{array}$ &. & \begin{tabular}{l|}
0 \\
0 \\
0
\end{tabular} & $\begin{array}{l}0 \\
0 \\
0\end{array}$ & j & \begin{tabular}{|l|}
0 \\
0 \\
\end{tabular} & $\begin{array}{l}0 \\
0 \\
0\end{array}$ & \begin{tabular}{l|l} 
\\
0 \\
0
\end{tabular} & 0 & & 0 & & & I & $\mathbf{M}$ & \\
\hline
\end{tabular}

\begin{tabular}{|c|c|c|c|c|c|c|c|c|c|c|c|c|c|c|c|c|c|c|c|c|c|c|c|c|c|c|c|c|c|c|c|c|c|c|c|c|}
\hline & $\begin{array}{l}0 \\
5 \\
5 \\
5\end{array}$ & $\begin{array}{l}0 \\
0\end{array}$ & $\begin{array}{l}1 \\
0\end{array}$ & $\begin{array}{l}1 \\
- \\
0\end{array}$ & $\begin{array}{l} \\
0 \\
0\end{array}$ & 0 & $\begin{array}{l}0 \\
0 \\
0\end{array}$ & 0 & $\begin{array}{l}0 \\
- \\
0\end{array}$ & i & 0 & 0 & 0 & 0 & 0 & $i_{0}$ & $\begin{array}{l}0 \\
- \\
0\end{array}$ & \begin{tabular}{l|}
0 \\
5 \\
5 \\
5
\end{tabular} & 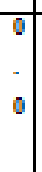 & $\begin{array}{l}0 \\
0 \\
0\end{array}$ & \begin{tabular}{l|}
0 \\
0 \\
0
\end{tabular} & $\begin{array}{l}0 \\
0 \\
0\end{array}$ & $\begin{array}{l}0 \\
0 \\
0\end{array}$ & $\begin{array}{l}0 \\
0 \\
0\end{array}$ & \begin{tabular}{l|l} 
\\
0 \\
0
\end{tabular} & $\begin{array}{l}0 \\
- \\
0\end{array}$ & $\begin{array}{l}0 \\
0 \\
0\end{array}$ & & \begin{tabular}{l|l} 
& \\
0 & \\
0
\end{tabular} & \begin{tabular}{l|l} 
& \\
- & \\
0 &
\end{tabular} & $\begin{array}{l} \\
- \\
0\end{array}$ & \begin{tabular}{l|l}
0 \\
0 \\
0
\end{tabular} & $\begin{array}{l} \\
0 \\
0\end{array}$ & \multicolumn{2}{|l|}{ I } & \\
\hline & $\begin{array}{l}0 \\
5 \\
5 \\
5\end{array}$ & $\begin{array}{l}0 \\
0\end{array}$ & $\begin{array}{l}1 \\
0 \\
0\end{array}$ & \begin{tabular}{l|l}
1 \\
. \\
0
\end{tabular} & $\begin{array}{l}0 \\
. \\
0\end{array}$ & j & $\begin{array}{l}1 \\
0 \\
0\end{array}$ & 0 & $\begin{array}{l}0 \\
- \\
0\end{array}$ & 0 & i & 0 & 0 & 0 & 0 & $\begin{array}{l}5 \\
5 \\
5\end{array}$ & $\begin{array}{l}0 \\
. \\
5 \\
5\end{array}$ & $\begin{array}{l}0 \\
5 \\
5 \\
5\end{array}$ & 1 & \begin{tabular}{l|}
0 \\
- \\
5 \\
5
\end{tabular} & $\begin{array}{l} \\
0 \\
0\end{array}$ & \begin{tabular}{l|}
1 \\
0 \\
0
\end{tabular} & $\begin{array}{l}0 \\
0 \\
0\end{array}$ & $\begin{array}{l}0 \\
0 \\
0\end{array}$ & $\begin{array}{l} \\
\\
0\end{array}$ & $\begin{array}{l}1 \\
0 \\
0\end{array}$ & $\begin{array}{l}0 \\
0 \\
0\end{array}$ & & \begin{tabular}{l|l} 
& \\
0 & \\
0
\end{tabular} & \begin{tabular}{l|l} 
& \\
. & \\
0 &
\end{tabular} & $\begin{array}{l}0 \\
0 \\
0\end{array}$ & \begin{tabular}{l|l} 
\\
0 \\
0
\end{tabular} & $\begin{array}{l}0 \\
0 \\
0\end{array}$ & \begin{tabular}{l|l}
$\mathrm{L}$ \\
\end{tabular} & - & 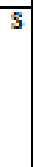 \\
\hline 8 & $\begin{array}{l}0 \\
5 \\
5 \\
5\end{array}$ & $\begin{array}{l}0 \\
0 \\
0\end{array}$ & $\begin{array}{l}1 \\
0\end{array}$ & \begin{tabular}{l|l}
1 \\
. \\
0
\end{tabular} & $\begin{array}{l} \\
0 \\
0\end{array}$ & $\begin{array}{l}0 \\
0 \\
0\end{array}$ & $\begin{array}{l}0 \\
0 \\
0\end{array}$ & $\begin{array}{l}0 \\
0 \\
0\end{array}$ & \begin{tabular}{|l|}
0 \\
- \\
0
\end{tabular} & ${ }_{0}$ & 0 & ${ }_{0}$ & 0 & 0 & $\begin{array}{l}0 \\
0 \\
0\end{array}$ & $\begin{array}{l} \\
5 \\
5 \\
5\end{array}$ & $\begin{array}{l}0 \\
- \\
5 \\
5\end{array}$ & $\begin{array}{l}0 \\
5 \\
5 \\
5\end{array}$ & 1 & $\begin{array}{l}0 \\
. \\
5 \\
5\end{array}$ & $\begin{array}{l}1 \\
0 \\
0\end{array}$ & $\begin{array}{l} \\
- \\
0\end{array}$ & $\begin{array}{l} \\
0 \\
0\end{array}$ & $\begin{array}{l}0 \\
- \\
0\end{array}$ & \begin{tabular}{l|l}
1 & \\
0 & \\
0
\end{tabular} & $\begin{array}{l}1 \\
0 \\
0\end{array}$ & \begin{tabular}{l|l} 
\\
0 \\
0
\end{tabular} & $\begin{array}{l}0 \\
0 \\
0\end{array}$ & \begin{tabular}{l|l} 
\\
0 \\
0
\end{tabular} & \begin{tabular}{l|l} 
& \\
. & \\
0 &
\end{tabular} & $\begin{array}{l}0 \\
- \\
0\end{array}$ & \begin{tabular}{l|l}
0 \\
0 \\
0
\end{tabular} & $\begin{array}{l}0 \\
0 \\
0\end{array}$ & \begin{tabular}{l|l} 
L \\
\end{tabular} & - & $s$ \\
\hline 9 & $\begin{array}{l}0 \\
5 \\
5 \\
5\end{array}$ & $\begin{array}{l}0 \\
0\end{array}$ & $\begin{array}{l}1 \\
0\end{array}$ & $\begin{array}{l}1 \\
0 \\
0\end{array}$ & $\begin{array}{l} \\
0 \\
0\end{array}$ & 0 & $\begin{array}{l}1 \\
0 \\
0\end{array}$ & 0 & $\begin{array}{l}0 \\
- \\
0\end{array}$ & 0 & i & 0 & 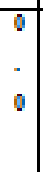 & i & i & $\begin{array}{l}0 \\
5 \\
5 \\
5\end{array}$ & $\begin{array}{l}0 \\
5 \\
5 \\
5\end{array}$ & $\begin{array}{l}0 \\
5 \\
5 \\
5\end{array}$ & 1 & $\begin{array}{l}0 \\
5 \\
5 \\
5\end{array}$ & $\begin{array}{l}1 \\
0 \\
0\end{array}$ & $\begin{array}{l}1 \\
- \\
0\end{array}$ & $\begin{array}{l} \\
0 \\
0\end{array}$ & $\begin{array}{l}0 \\
- \\
0\end{array}$ & $\begin{array}{l}1 \\
- \\
0\end{array}$ & $\begin{array}{l}1 \\
0\end{array}$ & $\begin{array}{l}0 \\
0 \\
0\end{array}$ & $\begin{array}{l}0 \\
0 \\
0\end{array}$ & \begin{tabular}{l|l}
0 \\
0 \\
0
\end{tabular} & $\begin{array}{l}0 \\
0 \\
0\end{array}$ & $\begin{array}{l}0 \\
- \\
0\end{array}$ & \begin{tabular}{l|l} 
& \\
0 & \\
0
\end{tabular} & $\begin{array}{l}0 \\
0 \\
0\end{array}$ & \begin{tabular}{l|l} 
L \\
\end{tabular} & - & $s$ \\
\hline$\overline{1}$ & \begin{tabular}{l|} 
\\
5 \\
5 \\
5
\end{tabular} & $\begin{array}{l}0 \\
- \\
0\end{array}$ & $\begin{array}{l}1 \\
- \\
0\end{array}$ & \begin{tabular}{|l|}
1 \\
0
\end{tabular} & $\begin{array}{l} \\
\\
0 \\
0\end{array}$ & 0 & $\begin{array}{l}1 \\
0\end{array}$ & 0 & $\begin{array}{l}0 \\
0 \\
0\end{array}$ & $\begin{array}{l}0 \\
0 \\
0\end{array}$ & $\begin{array}{l}0 \\
0\end{array}$ & $\begin{array}{l}0 \\
0 \\
0\end{array}$ & - & 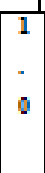 & o & : & $\begin{array}{l}0 \\
0 \\
5 \\
5\end{array}$ & \begin{tabular}{|l|}
0 \\
. \\
5 \\
5 \\
\end{tabular} & - & $\begin{array}{l}0 \\
5 \\
5 \\
5\end{array}$ & $\begin{array}{l}0 \\
0 \\
0\end{array}$ & \begin{tabular}{|l|}
1 \\
. \\
0
\end{tabular} & $\begin{array}{l}0 \\
0 \\
0\end{array}$ & $\begin{array}{l} \\
- \\
0\end{array}$ & $\begin{array}{l} \\
\\
0\end{array}$ & $\begin{array}{l}0 \\
0 \\
0\end{array}$ & $\begin{array}{l}0 \\
0 \\
0\end{array}$ & $\begin{array}{l} \\
- \\
0\end{array}$ & $\begin{array}{l}0 \\
0 \\
0\end{array}$ & $\begin{array}{l}0 \\
- \\
0\end{array}$ & \begin{tabular}{l|} 
\\
0 \\
0
\end{tabular} & \begin{tabular}{l|l}
0 \\
. \\
0
\end{tabular} & $\begin{array}{l}0 \\
0 \\
0\end{array}$ & $\overrightarrow{\mathrm{L}}$ & - & $s$ \\
\hline 1 & \begin{tabular}{l|}
0 \\
5 \\
5 \\
5
\end{tabular} & $\begin{array}{l}0 \\
5 \\
5 \\
5\end{array}$ & $\begin{array}{l}1 \\
0 \\
0\end{array}$ & \begin{tabular}{|l|} 
\\
- \\
0
\end{tabular} & $\begin{array}{l}1 \\
0 \\
0\end{array}$ & $\begin{array}{l}1 \\
0\end{array}$ & $\begin{array}{l}0 \\
0 \\
0\end{array}$ & 5 & $\begin{array}{l}0 \\
5 \\
5 \\
5\end{array}$ & $\begin{array}{l} \\
\\
0\end{array}$ & $\begin{array}{l}1 \\
0\end{array}$ & $\begin{array}{l}1 \\
0\end{array}$ & 0 & - & o & - & $\begin{array}{l}0 \\
0 \\
0\end{array}$ & $\begin{array}{l} \\
\\
0 \\
0\end{array}$ & 0 & \begin{tabular}{|l|}
0 \\
0 \\
\end{tabular} & $\begin{array}{l}0 \\
- \\
0\end{array}$ & $\begin{array}{l}0 \\
0 \\
0\end{array}$ & $\begin{array}{l}0 \\
0 \\
0\end{array}$ & $\begin{array}{l}0 \\
0 \\
0\end{array}$ & \begin{tabular}{l|l} 
\\
0 \\
0
\end{tabular} & $\begin{array}{l}0 \\
0 \\
0\end{array}$ & $\begin{array}{l}0 \\
0 \\
0\end{array}$ & $\begin{array}{l}0 \\
0 \\
0\end{array}$ & $\begin{array}{l}0 \\
0 \\
0\end{array}$ & $\begin{array}{l} \\
- \\
0\end{array}$ & $\begin{array}{l} \\
0 \\
0\end{array}$ & \begin{tabular}{l|l}
0 \\
0 \\
0
\end{tabular} & $\begin{array}{l}0 \\
0 \\
0\end{array}$ & $\mathrm{Y}$ & - & $s$ \\
\hline 2 & \begin{tabular}{l|}
0 \\
5 \\
5 \\
5
\end{tabular} & $\begin{array}{l}0 \\
5 \\
5 \\
5\end{array}$ & $\begin{array}{l}1 \\
- \\
0\end{array}$ & \begin{tabular}{|l|} 
\\
- \\
0
\end{tabular} & $\begin{array}{l}1 \\
0 \\
0\end{array}$ & $\begin{array}{l}0 \\
0\end{array}$ & $\begin{array}{l}0 \\
0\end{array}$ & 5 & $\begin{array}{l}0 \\
5 \\
5 \\
5\end{array}$ & $\begin{array}{l}1 \\
0 \\
0\end{array}$ & $\begin{array}{l}1 \\
0\end{array}$ & $\begin{array}{l}1 \\
0 \\
0\end{array}$ & - & $\begin{array}{l}0 \\
0 \\
0\end{array}$ & $\begin{array}{l}1 \\
0 \\
0\end{array}$ & $\begin{array}{l}0 \\
0 \\
0\end{array}$ & \begin{tabular}{|l|} 
\\
0 \\
0
\end{tabular} & $\begin{array}{l}0 \\
- \\
0\end{array}$ & 0 & \begin{tabular}{|l|}
0 \\
0 \\
\end{tabular} & $\begin{array}{l}0 \\
- \\
0\end{array}$ & $\begin{array}{l}0 \\
0 \\
0\end{array}$ & $\begin{array}{l}0 \\
0 \\
0\end{array}$ & $\begin{array}{l}0 \\
0 \\
0\end{array}$ & \begin{tabular}{l|}
0 \\
0 \\
0
\end{tabular} & $\begin{array}{l}0 \\
0 \\
0\end{array}$ & \begin{tabular}{l|l}
0 \\
0 \\
0
\end{tabular} & $\begin{array}{l}0 \\
0 \\
0\end{array}$ & & \begin{tabular}{l|l} 
\\
0 \\
0
\end{tabular} & $\begin{array}{l} \\
\\
0\end{array}$ & \begin{tabular}{l|l} 
& \\
0 & \\
0 &
\end{tabular} & $\begin{array}{l}0 \\
\\
0\end{array}$ & & - & s \\
\hline 1 & $\begin{array}{l}0 \\
5 \\
5 \\
5\end{array}$ & $\begin{array}{l}0 \\
5 \\
5 \\
5\end{array}$ & $\begin{array}{l}1 \\
- \\
0\end{array}$ & \begin{tabular}{|l|}
1 \\
0
\end{tabular} & $\begin{array}{l}1 \\
0 \\
0\end{array}$ & $\begin{array}{l}0 \\
0 \\
0\end{array}$ & $\begin{array}{l}0 \\
5 \\
5 \\
5\end{array}$ &. & $\begin{array}{l}0 \\
5 \\
5 \\
5\end{array}$ & $\begin{array}{l} \\
0 \\
0\end{array}$ & $\begin{array}{l}1 \\
0\end{array}$ & $\begin{array}{l}1 \\
0\end{array}$ & 0 & . & . & - & $\begin{array}{l}0 \\
- \\
0\end{array}$ & $\begin{array}{l}0 \\
0 \\
0\end{array}$ & $\begin{array}{l} \\
- \\
0\end{array}$ & \begin{tabular}{|l|}
0 \\
0 \\
\end{tabular} & $\begin{array}{l}0 \\
0 \\
0\end{array}$ & $\begin{array}{l} \\
. \\
0\end{array}$ & $\begin{array}{l} \\
0 \\
0\end{array}$ & $\begin{array}{l}0 \\
0 \\
0\end{array}$ & $\begin{array}{l}0 \\
0 \\
0\end{array}$ & $\begin{array}{l} \\
0 \\
0\end{array}$ & $\begin{array}{l} \\
\\
0\end{array}$ & $\begin{array}{l}0 \\
0\end{array}$ & & $\begin{array}{l} \\
\\
0\end{array}$ & $\begin{array}{l}0 \\
. \\
0\end{array}$ & \begin{tabular}{l|l}
0 & \\
0 & \\
0
\end{tabular} & $\begin{array}{l}0 \\
0 \\
0\end{array}$ & & - & s \\
\hline
\end{tabular}




\begin{tabular}{|c|c|c|c|c|c|c|c|c|c|c|c|c|c|c|c|c|c|c|c|c|c|c|c|c|c|c|c|c|c|c|c|c|c|c|c|c|}
\hline 4 & $\begin{array}{l}0 . \\
5\end{array}$ & $\begin{array}{l}0 \\
5 \\
5\end{array}$ & $\begin{array}{l}1 \\
- \\
0\end{array}$ & $\begin{array}{l}1 \\
- \\
0\end{array}$ & 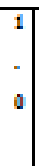 & 5 & $\begin{array}{l}5 \\
5\end{array}$ & -1 & 0 & 0 & - & 0 & 0 & 0 & - & 0 & $\begin{array}{l}0 \\
- \\
0\end{array}$ & $\begin{array}{l}0 \\
- \\
0\end{array}$ & - & \begin{tabular}{l|l|} 
& \\
- & \\
0 &
\end{tabular} & \begin{tabular}{l|l} 
& \\
- \\
0
\end{tabular} & $\begin{array}{l}0 \\
- \\
0\end{array}$ & - & $\begin{array}{l}0 \\
- \\
0\end{array}$ & \begin{tabular}{l|l} 
& \\
- & \\
0 &
\end{tabular} & \begin{tabular}{l|l} 
& \\
- & \\
0
\end{tabular} & - & - & $\begin{array}{l}0 \\
- \\
0\end{array}$ & - & $\begin{array}{l}0 \\
- \\
0\end{array}$ & $\begin{array}{l}0 \\
- \\
0\end{array}$ & $\begin{array}{l}0 \\
0 \\
0\end{array}$ & $\bar{Y}$ & - & s \\
\hline 1 & $\begin{array}{l}0 . \\
5\end{array}$ & $\begin{array}{l}0 \\
- \\
5 \\
5\end{array}$ & \begin{tabular}{l|}
1 \\
- \\
0
\end{tabular} & \begin{tabular}{l|}
1 \\
- \\
0
\end{tabular} & \begin{tabular}{l|}
1 \\
- \\
0
\end{tabular} & $\begin{array}{l}0 \\
0 \\
0\end{array}$ & $\begin{array}{l}0 \\
- \\
5 \\
5\end{array}$ & $\begin{array}{l} \\
- \\
5 \\
5\end{array}$ & $\begin{array}{l}0 \\
-5 \\
5 \\
5\end{array}$ & $\begin{array}{l}0 \\
- \\
0\end{array}$ & $\begin{array}{l}1 \\
- \\
0\end{array}$ & - & $\begin{array}{l}0 \\
- \\
0\end{array}$ & $\begin{array}{l}0 \\
- \\
0\end{array}$ & $\begin{array}{l}1 \\
- \\
0\end{array}$ & $\begin{array}{l}0 \\
- \\
0\end{array}$ & $\begin{array}{l}0 \\
0 \\
0\end{array}$ & $\begin{array}{l} \\
- \\
0\end{array}$ & $\begin{array}{l}0 \\
0 \\
0\end{array}$ & $\begin{array}{l} \\
- \\
0\end{array}$ & $\begin{array}{l}0 \\
0 \\
- \\
0\end{array}$ & $\begin{array}{l}0 \\
0 \\
0\end{array}$ & $\begin{array}{l}0 \\
0\end{array}$ & $\begin{array}{l}0 \\
0 \\
0\end{array}$ & \begin{tabular}{l|l} 
& \\
- & \\
0 &
\end{tabular} & \begin{tabular}{l|l}
0 \\
- \\
0
\end{tabular} & $\begin{array}{l}0 \\
0 \\
0\end{array}$ & $\begin{array}{l}0 \\
0 \\
0\end{array}$ & $\begin{array}{l}0 \\
- \\
0\end{array}$ & $\begin{array}{l}0 \\
0 \\
0\end{array}$ & $\begin{array}{l}0 \\
- \\
0\end{array}$ & $\begin{array}{l}0 \\
0\end{array}$ & \begin{tabular}{l|}
0 \\
- \\
0
\end{tabular} & $\bar{Y}$ & & $1-$ \\
\hline 1 & $\begin{array}{l}0 . \\
5 \\
5\end{array}$ & $\begin{array}{l}0 \\
- \\
5 \\
5\end{array}$ & \begin{tabular}{l|}
1 \\
- \\
0
\end{tabular} & \begin{tabular}{l|}
1 \\
- \\
0
\end{tabular} & \begin{tabular}{|l|}
1 \\
- \\
0
\end{tabular} & $\begin{array}{l}0 \\
- \\
0\end{array}$ & \begin{tabular}{l|}
0 \\
- \\
0
\end{tabular} & $\begin{array}{l}0 \\
- \\
5\end{array}$ & $\begin{array}{l}- \\
5 \\
5\end{array}$ & $\begin{array}{l} \\
- \\
0\end{array}$ & - & $\begin{array}{l} \\
- \\
0\end{array}$ & 0 & ${ }_{0}^{-}$ & - & - & $\begin{array}{l}0 \\
0 \\
0\end{array}$ & \begin{tabular}{l|}
0 \\
- \\
0
\end{tabular} & $\begin{array}{l}0 \\
0\end{array}$ & $\begin{array}{l}0 \\
- \\
0\end{array}$ & \begin{tabular}{l|}
0 \\
- \\
0
\end{tabular} & $\begin{array}{l}0 \\
- \\
0\end{array}$ & $\begin{array}{l}0 \\
0\end{array}$ & $\begin{array}{l} \\
0 \\
0\end{array}$ & \begin{tabular}{l|l} 
& \\
- & \\
0 &
\end{tabular} & \begin{tabular}{l|l}
0 \\
- \\
0
\end{tabular} & $\begin{array}{l}0 \\
0 \\
0\end{array}$ & $\begin{array}{l}0 \\
0 \\
0\end{array}$ & $\begin{array}{l}0 \\
0 \\
0\end{array}$ & $\begin{array}{l}0 \\
0 \\
0\end{array}$ & $\begin{array}{l}0 \\
- \\
0\end{array}$ & $\begin{array}{l}0 \\
0 \\
0\end{array}$ & $\begin{array}{l}0 \\
- \\
0\end{array}$ & $\bar{Y}$ & $\bar{M}$ & \begin{tabular}{l|l}
-
\end{tabular} \\
\hline$\overline{1}$ & $\begin{array}{l}0 . \\
5\end{array}$ & $\begin{array}{l}0 \\
5 \\
5 \\
5\end{array}$ & \begin{tabular}{l|}
1 \\
- \\
0
\end{tabular} & \begin{tabular}{l|}
1 \\
- \\
0
\end{tabular} & \begin{tabular}{l|}
1 \\
- \\
0
\end{tabular} & $\begin{array}{l}0 \\
0 \\
0\end{array}$ & $\begin{array}{l}0 \\
- \\
5 \\
5\end{array}$ & 5 & 0 & $\begin{array}{l}0 \\
- \\
0\end{array}$ & $\begin{array}{l}0 \\
- \\
0\end{array}$ & 0 & - & 0 & - & - & $\begin{array}{l}0 \\
0 \\
0\end{array}$ & $\begin{array}{l}0 \\
0 \\
0\end{array}$ & - & $\begin{array}{l}0 \\
- \\
0\end{array}$ & \begin{tabular}{l|l} 
& \\
- & \\
0 &
\end{tabular} & $\begin{array}{l}0 \\
0 \\
0\end{array}$ & - & $\begin{array}{l} \\
- \\
0\end{array}$ & $\begin{array}{l} \\
- \\
0\end{array}$ & \begin{tabular}{l|l} 
& \\
- & \\
0 &
\end{tabular} & $\begin{array}{l}0 \\
0 \\
0\end{array}$ & $\begin{array}{l}0 \\
- \\
0\end{array}$ & $\begin{array}{l}0 \\
0 \\
0\end{array}$ & $\begin{array}{l}0 \\
0 \\
0\end{array}$ & $\begin{array}{l}0 \\
- \\
0\end{array}$ & $\begin{array}{l}0 \\
0 \\
0\end{array}$ & $\begin{array}{l}0 \\
- \\
0\end{array}$ & $\bar{Y}$ & $\bar{M}$ & $1-$ \\
\hline 1 & $\begin{array}{l}0 . \\
5\end{array}$ & $\begin{array}{l}0 \\
5 \\
5 \\
5\end{array}$ & \begin{tabular}{l|}
1 \\
- \\
0
\end{tabular} & $\begin{array}{l}1 \\
- \\
0\end{array}$ & \begin{tabular}{l|}
1 \\
- \\
0
\end{tabular} & $\begin{array}{l}0 \\
- \\
0\end{array}$ & $\begin{array}{l}0 \\
-5 \\
5 \\
5\end{array}$ & 0 & $\begin{array}{l}0 \\
0 \\
0\end{array}$ & $\begin{array}{l} \\
- \\
0\end{array}$ & - & 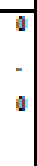 & $\begin{array}{l}0 \\
- \\
0\end{array}$ & 0 & $\begin{array}{l}0 \\
-\end{array}$ & $\begin{array}{l}0 \\
-\end{array}$ & $\begin{array}{l}0 \\
0 \\
0\end{array}$ & $\begin{array}{l}0 \\
- \\
0\end{array}$ & $\begin{array}{l}0 \\
0 \\
0\end{array}$ & $\begin{array}{l}0 \\
0 \\
0\end{array}$ & $\begin{array}{l}0 \\
- \\
0\end{array}$ & $\begin{array}{l}0 \\
0 \\
0\end{array}$ & $\begin{array}{l}0 \\
0\end{array}$ & $\begin{array}{l}0 \\
- \\
0\end{array}$ & $\begin{array}{l}0 \\
0 \\
0\end{array}$ & $\begin{array}{l} \\
0 \\
0\end{array}$ & $\begin{array}{l}0 \\
0 \\
0\end{array}$ & $\begin{array}{l}0 \\
- \\
0\end{array}$ & $\begin{array}{l}0 \\
- \\
0\end{array}$ & $\begin{array}{l}0 \\
- \\
0\end{array}$ & $\begin{array}{l}0 \\
- \\
0\end{array}$ & $\begin{array}{l}0 \\
0 \\
0\end{array}$ & $\begin{array}{l}0 \\
0 \\
0\end{array}$ & $\bar{Y}$ & $\bar{M}$ & i. \\
\hline 1 & $\begin{array}{l}0 . \\
5 \\
5\end{array}$ & $\begin{array}{l}0 \\
- \\
5 \\
5\end{array}$ & $\begin{array}{l}1 \\
- \\
0\end{array}$ & $\begin{array}{l}1 \\
- \\
0\end{array}$ & \begin{tabular}{l|}
1 \\
- \\
0
\end{tabular} & $\begin{array}{l}0 \\
- \\
0\end{array}$ & $\begin{array}{l} \\
- \\
0\end{array}$ & 0 & 0 & $\begin{array}{l}0 \\
0 \\
0\end{array}$ & - & 0 & $\begin{array}{l}0 \\
- \\
0\end{array}$ & 0 & $\begin{array}{l}0 \\
- \\
0\end{array}$ & 0 & $\begin{array}{l}0 \\
- \\
0\end{array}$ & $\begin{array}{l}0 \\
- \\
0\end{array}$ & 0 & $\begin{array}{l}0 \\
0 \\
0\end{array}$ & $\begin{array}{l}0 \\
- \\
0\end{array}$ & 0 & $\begin{array}{l}0 \\
0 \\
0\end{array}$ & $\begin{array}{l}0 \\
0 \\
0\end{array}$ & $\begin{array}{l}0 \\
- \\
0\end{array}$ & $\begin{array}{l} \\
- \\
0\end{array}$ & $\begin{array}{l}0 \\
0 \\
0\end{array}$ & $\begin{array}{l}0 \\
0 \\
0\end{array}$ & $\begin{array}{l}0 \\
0 \\
0\end{array}$ & $\begin{array}{l}0 \\
0 \\
0\end{array}$ & $\begin{array}{l}0 \\
- \\
0\end{array}$ & $\begin{array}{l}0 \\
0 \\
0\end{array}$ & $\begin{array}{l}0 \\
0 \\
0\end{array}$ & $\bar{Y}$ & - & - \\
\hline 0 & $\begin{array}{l}5 \\
5\end{array}$ & $\begin{array}{l}0 \\
5 \\
5 \\
5\end{array}$ & 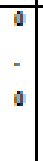 & - & 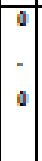 & 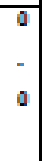 &. & 0 & 0 & 0 & $=$ & 0 &. & 0 & $=$ & - & $\begin{array}{l}0 \\
- \\
0\end{array}$ & $\begin{array}{l} \\
- \\
0\end{array}$ & - & $\begin{array}{l}0 \\
0 \\
0\end{array}$ & $\begin{array}{l}0 \\
- \\
0\end{array}$ & - & - & 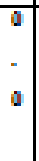 & $\begin{array}{l}0 \\
- \\
0\end{array}$ & $\begin{array}{l} \\
- \\
0\end{array}$ & - & - & $\begin{array}{l}0 \\
0 \\
0\end{array}$ & $\begin{array}{l}0 \\
0 \\
0\end{array}$ & $\begin{array}{l}0 \\
- \\
0\end{array}$ & $\begin{array}{l}0 \\
0 \\
0\end{array}$ & $\begin{array}{l}0 \\
0 \\
0\end{array}$ & $\mathrm{~T}$ & - & - \\
\hline 1 & $\begin{array}{l}0 . \\
5 \\
5\end{array}$ & $\begin{array}{l}0 \\
5 \\
5 \\
5\end{array}$ & $\begin{array}{l}0 \\
0 \\
0\end{array}$ & $\begin{array}{l}0 \\
- \\
0\end{array}$ & 0 & ${ }_{0}^{-}$ & 0 & 0 & 0 &. & 0 & 0 & $\begin{array}{l}- \\
5 \\
5\end{array}$ & 0 & 0 & 5 & 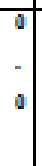 & $\begin{array}{l}0 \\
- \\
0\end{array}$ & 5 & $\begin{array}{l}0 \\
0 \\
0\end{array}$ & $\begin{array}{l}0 \\
- \\
0\end{array}$ & 0 & 0 & $\begin{array}{l}0 \\
- \\
0\end{array}$ & $\begin{array}{l}0 \\
- \\
0\end{array}$ & $\begin{array}{l} \\
- \\
0\end{array}$ & 0 & - & $\begin{array}{l}0 \\
5 \\
5\end{array}$ & $\begin{array}{l}0 \\
0 \\
0\end{array}$ & $\begin{array}{l}0 \\
- \\
0\end{array}$ & $\begin{array}{l}0 \\
0 \\
0\end{array}$ & - & $\mathrm{T}$ & $\mathrm{M}$ & I. \\
\hline$\overline{2}$ & $\begin{array}{l}0 . \\
5 \\
5\end{array}$ & $\begin{array}{l}0 \\
5 \\
5 \\
5\end{array}$ & $\begin{array}{l}0 \\
- \\
0\end{array}$ & 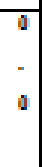 & o & $\begin{array}{l}0 \\
- \\
0\end{array}$ & $\begin{array}{l}0 \\
- \\
0\end{array}$ & - & $\begin{array}{l}0 \\
- \\
0\end{array}$ & $\begin{array}{l}0 \\
- \\
0\end{array}$ & 0 & - & $\begin{array}{l}0 \\
5 \\
5 \\
5\end{array}$ & 5 & $\begin{array}{l}0 \\
- \\
0\end{array}$ & i & 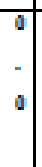 & $\begin{array}{l} \\
- \\
0\end{array}$ & 5 & $\begin{array}{l}0 \\
0 \\
0\end{array}$ & $\begin{array}{l}0 \\
- \\
0\end{array}$ & 0 & $\overline{0}$ & $\begin{array}{l}- \\
0\end{array}$ & $\begin{array}{l}0 \\
- \\
0\end{array}$ & $\begin{array}{l}0 \\
- \\
0\end{array}$ & $\bar{c}^{-}$ & - & $\begin{array}{l}0 \\
5 \\
5\end{array}$ & $\begin{array}{l}0 \\
0 \\
0\end{array}$ & $\begin{array}{l}0 \\
0 \\
0\end{array}$ & $\begin{array}{l}0 \\
0 \\
0\end{array}$ & $\begin{array}{l}0 \\
0 \\
0\end{array}$ & $\mathrm{~T}$ & $\bar{M}$ & $1-$ \\
\hline 3 & $\begin{array}{l}0 . \\
5 \\
5\end{array}$ & $\begin{array}{l}0 \\
5 \\
5 \\
5\end{array}$ & - & $\begin{array}{l}0 \\
- \\
0\end{array}$ &. & - &. & $:$ & 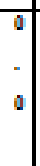 &. & - & 0 & $\begin{array}{l}- \\
5 \\
5\end{array}$ & 0 & ${ }_{0}$ & 5 & $\begin{array}{l}0 \\
- \\
0\end{array}$ & $\begin{array}{l}0 \\
- \\
0\end{array}$ & 0 & $\begin{array}{l}0 \\
- \\
0\end{array}$ & $\begin{array}{l}0 \\
- \\
0\end{array}$ & $\begin{array}{l}0 \\
0 \\
0\end{array}$ & - & 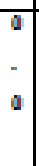 & $\begin{array}{l}0 \\
- \\
0\end{array}$ & $\begin{array}{l}0 \\
- \\
0\end{array}$ & - & - & $\begin{array}{l}0 \\
5 \\
5\end{array}$ & - & $\begin{array}{l}0 \\
0 \\
0\end{array}$ & $\begin{array}{l}0 \\
0 \\
0\end{array}$ & - & $\mathrm{T}$ & & $1-$ \\
\hline 4 & $\begin{array}{l}0 . \\
5 \\
5\end{array}$ & $\begin{array}{l}0 \\
5 \\
5 \\
5\end{array}$ & 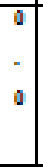 & -1 & $=$ & -1 & 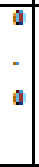 & 0 & 0 & $=$ & 0 & - & $\begin{array}{l}- \\
5 \\
5\end{array}$ & 5 & $\begin{array}{l}- \\
0\end{array}$ & 5 & 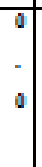 & $\begin{array}{l}0 \\
- \\
0\end{array}$ & 5 & $\begin{array}{l}0 \\
0 \\
0\end{array}$ & $\begin{array}{l}0 \\
- \\
0\end{array}$ & 0 & ; & 0 & $\begin{array}{l}0 \\
- \\
0\end{array}$ & $\begin{array}{l} \\
- \\
0\end{array}$ & - & - & - & $\begin{array}{l}0 \\
0 \\
0\end{array}$ & $\begin{array}{l}0 \\
0 \\
0\end{array}$ & $\begin{array}{l}0 \\
0 \\
0\end{array}$ & $\begin{array}{l}0 \\
0 \\
0\end{array}$ & $\bar{T}$ & & $1-$ \\
\hline 5 & $\begin{array}{l}0 . \\
5\end{array}$ & $\begin{array}{l}0 \\
5 \\
5 \\
5\end{array}$ & - & $\begin{array}{l}0 \\
0\end{array}$ & $\begin{array}{l}0 \\
- \\
0\end{array}$ & $\begin{array}{l}0 \\
- \\
0\end{array}$ & $\begin{array}{l}0 \\
- \\
0\end{array}$ & $\begin{array}{l}0 \\
0 \\
0\end{array}$ & $\begin{array}{l}0 \\
- \\
0\end{array}$ & $\begin{array}{l}0 \\
- \\
0\end{array}$ & - & 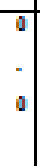 & $\begin{array}{l}0 \\
5 \\
5 \\
5\end{array}$ & $\begin{array}{l}- \\
5\end{array}$ & $\begin{array}{l}0 \\
- \\
0\end{array}$ & 5 & $\begin{array}{l}0 \\
- \\
0\end{array}$ & $\begin{array}{l} \\
- \\
0\end{array}$ & 5 & $\begin{array}{l}0 \\
0 \\
0\end{array}$ & $\begin{array}{l}0 \\
0 \\
0\end{array}$ & $\begin{array}{l}0 \\
0 \\
0\end{array}$ & $\begin{array}{l}0 \\
0 \\
0\end{array}$ & $\begin{array}{l}0 \\
- \\
0\end{array}$ & $\begin{array}{l}0 \\
- \\
0\end{array}$ & $\begin{array}{l} \\
- \\
0\end{array}$ & - & $\begin{array}{l}0 \\
0 \\
0\end{array}$ & $\begin{array}{l}0 \\
- \\
5 \\
5\end{array}$ & $\begin{array}{l}0 \\
0 \\
0\end{array}$ & $\begin{array}{l}0 \\
0 \\
0\end{array}$ & $\begin{array}{l}0 \\
0 \\
0\end{array}$ & $\begin{array}{l}0 \\
0 \\
0\end{array}$ & $\bar{T}$ & & $1-$ \\
\hline 6 & $\begin{array}{l}0 . \\
5 \\
5\end{array}$ & $\begin{array}{l}0 \\
5 \\
5 \\
5\end{array}$ & - & 0 & $\begin{array}{l}0 \\
- \\
0\end{array}$ & $\dot{0}_{0}$ & 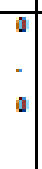 & -1 & - & - & - & 0 & $\begin{array}{l}0 \\
- \\
0\end{array}$ & $\begin{array}{l}5 \\
5\end{array}$ & - & $\begin{array}{l}- \\
5 \\
5\end{array}$ & $\begin{array}{l}0 \\
- \\
0\end{array}$ & $\begin{array}{l}0 \\
- \\
0\end{array}$ & $\begin{array}{l}5 \\
5 \\
5\end{array}$ & $\begin{array}{l}0 \\
0 \\
0\end{array}$ & $\begin{array}{l}0 \\
- \\
0\end{array}$ & $\begin{array}{l}0 \\
0 \\
0\end{array}$ & - & 0 & $\begin{array}{l}0 \\
- \\
0\end{array}$ & $\begin{array}{l}0 \\
0 \\
0\end{array}$ & $\begin{array}{l}0 \\
0 \\
0\end{array}$ & - & $\begin{array}{l}0 \\
5 \\
5 \\
5\end{array}$ & $\begin{array}{l}0 \\
0 \\
0\end{array}$ & $\begin{array}{l}0 \\
0 \\
0\end{array}$ & $\begin{array}{l}0 \\
0 \\
0\end{array}$ & - & $\mathrm{T}$ & & $1-$ \\
\hline 7 & $\begin{array}{l}0 . \\
5 \\
5\end{array}$ & 5 & - & 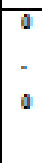 & 0 & - & 0 & 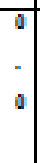 & 0 & 0 & 0 & 0 & 0 & 0 & 0 & 0 & $\begin{array}{l}0 \\
0\end{array}$ & $\begin{array}{l}0 \\
- \\
0\end{array}$ & $\begin{array}{l}0 \\
0 \\
0\end{array}$ & $\begin{array}{l}0 \\
0 \\
0\end{array}$ & $\begin{array}{l}0 \\
- \\
0\end{array}$ & $\begin{array}{l}0 \\
0 \\
0\end{array}$ & - & 0 & $\begin{array}{l}0 \\
- \\
0\end{array}$ & $\begin{array}{l} \\
0 \\
0\end{array}$ & j & - & 5 & $\begin{array}{l}0 \\
0 \\
0\end{array}$ & $\begin{array}{l}0 \\
0 \\
0\end{array}$ & $\begin{array}{l}0 \\
0 \\
0\end{array}$ & $\begin{array}{l}0 \\
0 \\
0\end{array}$ & $\mathrm{~T}$ & & If \\
\hline
\end{tabular}




\begin{tabular}{|c|c|c|c|c|c|c|c|c|c|c|c|c|c|c|c|c|c|c|c|c|c|c|c|c|c|c|c|c|c|c|c|c|c|c|c|c|c|}
\hline 8 & $\begin{array}{l}0 . \\
5\end{array}$ & $\begin{array}{l}- \\
5 \\
5\end{array}$ & 0 & - & 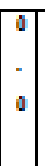 & $\begin{array}{l}0 \\
0 \\
0\end{array}$ & - & 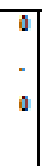 & 0 & 0 & 0 & 0 & 0 & 0 & \begin{tabular}{l|l} 
& \\
- \\
0
\end{tabular} & \begin{tabular}{l|} 
\\
- \\
5 \\
5
\end{tabular} & \begin{tabular}{l|} 
\\
- \\
0
\end{tabular} & $\begin{array}{l}0 \\
- \\
0\end{array}$ & $\begin{array}{l}0 \\
- \\
5 \\
5\end{array}$ & \begin{tabular}{|l|}
0 \\
- \\
0
\end{tabular} & \begin{tabular}{l|l} 
& \\
- \\
0
\end{tabular} & \begin{tabular}{l|}
0 \\
- \\
0
\end{tabular} & \begin{tabular}{|l|}
0 \\
- \\
0
\end{tabular} & \begin{tabular}{|l|}
0 \\
- \\
0
\end{tabular} & \begin{tabular}{l|l} 
& \\
- & \\
0 &
\end{tabular} & \begin{tabular}{|l|}
0 \\
- \\
0
\end{tabular} & $\begin{array}{l}1 \\
- \\
0\end{array}$ & \begin{tabular}{|l|}
0 \\
- \\
0
\end{tabular} & $\begin{array}{l}0 \\
-5 \\
5 \\
5\end{array}$ & - & $\begin{array}{l}0 \\
0\end{array}$ & $\begin{array}{l}0 \\
0 \\
0\end{array}$ & & & & & 5 \\
\hline 2 & $\begin{array}{l}0 . \\
5 \\
5\end{array}$ & $\begin{array}{l}0 \\
5 \\
5\end{array}$ & - & $\begin{array}{l}0 \\
- \\
0\end{array}$ & $\begin{array}{l}0 \\
- \\
0\end{array}$ & $\begin{array}{l}0 \\
- \\
0\end{array}$ & $\begin{array}{l} \\
- \\
0\end{array}$ & \begin{tabular}{|l|}
0 \\
\end{tabular} & $\begin{array}{l}0 \\
- \\
0\end{array}$ & 0 & $\begin{array}{l}0 \\
- \\
0\end{array}$ & - & 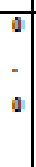 & - & $\begin{array}{l}0 \\
- \\
0\end{array}$ & $\begin{array}{l}0 \\
- \\
5 \\
5\end{array}$ & $\begin{array}{l} \\
- \\
0\end{array}$ & $\begin{array}{l} \\
0 \\
0\end{array}$ & $\begin{array}{l}0 \\
- \\
0\end{array}$ & $\begin{array}{l}0 \\
- \\
0\end{array}$ & $\begin{array}{l} \\
- \\
0\end{array}$ & -1 & \begin{tabular}{|l|} 
\\
- \\
0
\end{tabular} & \begin{tabular}{l|l|} 
& \\
- \\
0
\end{tabular} & \begin{tabular}{l|}
0 \\
- \\
0
\end{tabular} & - & $\begin{array}{l}0 \\
- \\
0\end{array}$ & $\begin{array}{l}1 \\
- \\
0\end{array}$ & $\begin{array}{l}0 \\
- \\
5 \\
5\end{array}$ & - & $\begin{array}{l}0 \\
0\end{array}$ & $\begin{array}{l}0 \\
- \\
0\end{array}$ & & & & & 5 \\
\hline 3 & $\begin{array}{l}0 . \\
5 \\
5\end{array}$ & $\begin{array}{l}0 \\
5 \\
5\end{array}$ & $\begin{array}{l}0 \\
- \\
0\end{array}$ & - & $\begin{array}{l}0 \\
- \\
0\end{array}$ & 00 & $\begin{array}{l}0 \\
- \\
0\end{array}$ & \begin{tabular}{|l|}
0 \\
\end{tabular} & $\begin{array}{l}0 \\
- \\
0\end{array}$ & 0 & - & 0 & 0 & - & $\begin{array}{l} \\
- \\
0\end{array}$ & $\begin{array}{l}0 \\
0 \\
0\end{array}$ & \begin{tabular}{l|}
0 \\
- \\
0
\end{tabular} & $\begin{array}{l}0 \\
0 \\
0\end{array}$ & $\begin{array}{l}0 \\
0 \\
0\end{array}$ & \begin{tabular}{|l|} 
\\
0 \\
0
\end{tabular} & $\begin{array}{l} \\
- \\
0\end{array}$ & 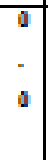 & \begin{tabular}{|l|} 
\\
- \\
0
\end{tabular} & \begin{tabular}{l|l|} 
& \\
- \\
0
\end{tabular} & \begin{tabular}{l|}
0 \\
- \\
0
\end{tabular} & - & $\begin{array}{l}1 \\
- \\
0\end{array}$ & $\begin{array}{l}0 \\
- \\
0\end{array}$ & $\begin{array}{l}0 \\
- \\
5 \\
5\end{array}$ & $\begin{array}{l}0 \\
0 \\
\end{array}$ & $\begin{array}{l}0 \\
0 \\
0\end{array}$ & $\begin{array}{l}0 \\
- \\
0\end{array}$ & $\begin{array}{l}0 \\
0 \\
0\end{array}$ & 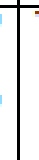 & & & 5 \\
\hline 3 & $\begin{array}{l}0 . \\
5 \\
5\end{array}$ & $\begin{array}{l}0 \\
5 \\
5 \\
5\end{array}$ & ${ }_{0}$ & $\begin{array}{l}0 \\
- \\
0\end{array}$ & $\begin{array}{l} \\
- \\
0\end{array}$ & 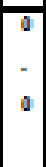 & $\begin{array}{l}0 \\
- \\
0\end{array}$ & \begin{tabular}{|l|}
0 \\
\end{tabular} & $\begin{array}{l}0 \\
0 \\
0\end{array}$ & $\begin{array}{l}- \\
0\end{array}$ & - & 0 & - & - & $\begin{array}{l}0 \\
- \\
0\end{array}$ & $\begin{array}{l}0 \\
5 \\
5 \\
5\end{array}$ & \begin{tabular}{l|l} 
& \\
- \\
0
\end{tabular} & $\begin{array}{l}0 \\
0 \\
0\end{array}$ & $\begin{array}{l}0 \\
5 \\
5 \\
5\end{array}$ & $\begin{array}{l}0 \\
- \\
0\end{array}$ & \begin{tabular}{l|l} 
& \\
- \\
0
\end{tabular} & -1 & $\begin{array}{l}0 \\
- \\
0\end{array}$ & $\begin{array}{l}0 \\
- \\
0\end{array}$ & \begin{tabular}{l|l} 
& \\
- & \\
0 &
\end{tabular} & $\begin{array}{l}0 \\
0\end{array}$ & \begin{tabular}{|l}
1 \\
- \\
0
\end{tabular} & $\begin{array}{l}1 \\
- \\
0\end{array}$ & $\begin{array}{l}- \\
5 \\
5\end{array}$ & - & $\begin{array}{l}0 \\
- \\
0\end{array}$ & $\begin{array}{l}0 \\
- \\
0\end{array}$ & & & & & $\overline{8}$ \\
\hline 3 & $\begin{array}{l}0 . \\
5\end{array}$ & $\begin{array}{l}0 \\
5 \\
5\end{array}$ & 0 & $\begin{array}{l}1 \\
- \\
0\end{array}$ & $\begin{array}{l}0 \\
- \\
0\end{array}$ & - & $\begin{array}{l}0 \\
- \\
0\end{array}$ & 0 & $\begin{array}{l}0 \\
- \\
0\end{array}$ & $\begin{array}{ll}0 \\
\end{array}$ & 0 & 0 & 0 & 0 & $\begin{array}{l}0 \\
- \\
0\end{array}$ & $\begin{array}{l}0 \\
0 \\
0\end{array}$ & $\begin{array}{l}0 \\
- \\
0\end{array}$ & $\begin{array}{l}0 \\
\end{array}$ & $\begin{array}{l}0 \\
- \\
0\end{array}$ & $\begin{array}{l}0 \\
- \\
0\end{array}$ & $\begin{array}{l}0 \\
- \\
0\end{array}$ & 0 & \begin{tabular}{|l}
0 \\
- \\
0
\end{tabular} & $\begin{array}{l} \\
- \\
0\end{array}$ & \begin{tabular}{l|} 
\\
- \\
0
\end{tabular} & $\begin{array}{l}0 \\
0 \\
0\end{array}$ & $\begin{array}{l}0 \\
- \\
0\end{array}$ & $\begin{array}{l}0 \\
0 \\
0\end{array}$ & $\begin{array}{l}0 \\
- \\
0\end{array}$ & - & $\begin{array}{l}0 \\
- \\
0\end{array}$ & $\begin{array}{l}0 \\
0\end{array}$ & & & & & $\cdot$ \\
\hline 3 & $\begin{array}{l}0 . \\
5 \\
5\end{array}$ & $\begin{array}{l}0 \\
5 \\
5\end{array}$ & 0 & $\begin{array}{l}1 \\
-\end{array}$ & $\begin{array}{l}0 \\
- \\
0\end{array}$ & $\begin{array}{l}0 \\
- \\
0\end{array}$ & $\begin{array}{l}0 \\
- \\
0\end{array}$ & 0 & $\begin{array}{l}0 \\
- \\
0\end{array}$ & \begin{tabular}{|l|}
0 \\
\end{tabular} & $\begin{array}{l}0 \\
0\end{array}$ & 0 & $\begin{array}{l}0 \\
-\end{array}$ & 0 & $\begin{array}{l}0 \\
0 \\
0\end{array}$ & $\begin{array}{l}0 \\
5 \\
5 \\
5\end{array}$ & $\begin{array}{l}0 \\
- \\
0\end{array}$ & $\begin{array}{l}0 \\
- \\
5 \\
5\end{array}$ & $\begin{array}{l}0 \\
- \\
0\end{array}$ & \begin{tabular}{|l|}
0 \\
-
\end{tabular} & $\begin{array}{l} \\
- \\
0\end{array}$ & 0 & $\begin{array}{l}0 \\
5 \\
5 \\
5\end{array}$ & $\begin{array}{l} \\
0 \\
0\end{array}$ & $\begin{array}{l}0 \\
- \\
0\end{array}$ & $\begin{array}{l}0 \\
0\end{array}$ & $\begin{array}{l}0 \\
- \\
0\end{array}$ & $\begin{array}{l}0 \\
0 \\
0\end{array}$ & $\begin{array}{l}0 \\
- \\
0\end{array}$ & $\begin{array}{l} \\
0 \\
\end{array}$ & $\begin{array}{l}0 \\
0 \\
0\end{array}$ & $\begin{array}{l}0 \\
0 \\
0\end{array}$ & & & & & 5 \\
\hline 4 & $\begin{array}{l}0 . \\
5 \\
5\end{array}$ & $\begin{array}{l}0 \\
5 \\
5\end{array}$ & ${ }_{0}^{-}$ & $\begin{array}{l}1 \\
-\end{array}$ & 0 & - & $\begin{array}{l} \\
- \\
0\end{array}$ & 0 & $\begin{array}{l}0 \\
- \\
0\end{array}$ & \begin{tabular}{|l|}
1 \\
\end{tabular} & - & 0 & 0 & 5 & $\begin{array}{l}0 \\
- \\
0\end{array}$ & $\begin{array}{l}0 \\
0 \\
0\end{array}$ & $\begin{array}{l}0 \\
- \\
0\end{array}$ & $\begin{array}{l}0 \\
-\end{array}$ & $\begin{array}{l}0 \\
- \\
0\end{array}$ & $\begin{array}{l}0 \\
- \\
5 \\
5\end{array}$ & $\begin{array}{l}0 \\
- \\
0\end{array}$ & \begin{tabular}{|l|}
0 \\
\end{tabular} & $\begin{array}{l}0 \\
\\
0\end{array}$ & \begin{tabular}{l|l} 
& \\
5 & \\
5 & \\
5 &
\end{tabular} & $\begin{array}{l}0 \\
- \\
0\end{array}$ & - & $\begin{array}{l}0 \\
- \\
0\end{array}$ & $\begin{array}{l}0 \\
0 \\
0\end{array}$ & $\begin{array}{l}0 \\
- \\
0\end{array}$ & $\begin{array}{l}0 \\
0\end{array}$ & $\begin{array}{l}0 \\
0 \\
0\end{array}$ & $\begin{array}{l}0 \\
- \\
0\end{array}$ & & & & & 5 \\
\hline 5 & $\begin{array}{l}0 . \\
5 \\
5\end{array}$ & $\begin{array}{l}0 \\
5 \\
5\end{array}$ & - & : & $\begin{array}{l} \\
- \\
0\end{array}$ & $\begin{array}{l}0 \\
- \\
0\end{array}$ & $\begin{array}{l} \\
- \\
0\end{array}$ & 0 & $\begin{array}{l}0 \\
- \\
0\end{array}$ & 0 & $\begin{array}{l}0 \\
- \\
0\end{array}$ & 0 & 0 & $\begin{array}{l}0 \\
0\end{array}$ & $\begin{array}{l}0 \\
0 \\
0\end{array}$ & $\begin{array}{l}0 \\
5 \\
5 \\
5\end{array}$ & $\begin{array}{l} \\
0 \\
0\end{array}$ & $\begin{array}{l}0 \\
5 \\
5 \\
5\end{array}$ & $\begin{array}{l}0 \\
- \\
0\end{array}$ & $\begin{array}{l}0 \\
5 \\
5 \\
5\end{array}$ & $\begin{array}{l}0 \\
0 \\
0\end{array}$ & $\begin{array}{l}- \\
0\end{array}$ & $\begin{array}{l}5 \\
5 \\
5\end{array}$ & \begin{tabular}{|l|}
0 \\
5 \\
5 \\
5 \\
\end{tabular} & $\begin{array}{l}0 \\
0 \\
0\end{array}$ & - & $\begin{array}{l}0 \\
- \\
0\end{array}$ & $\begin{array}{l}0 \\
0 \\
0\end{array}$ & $\begin{array}{l}0 \\
- \\
0\end{array}$ & $\begin{array}{l} \\
\end{array}$ & $\begin{array}{l}0 \\
- \\
0\end{array}$ & $\begin{array}{l}0 \\
- \\
0\end{array}$ & & & & & 5 \\
\hline$\sqrt{3}$ & $\begin{array}{l}0 . \\
5 \\
5\end{array}$ & $\begin{array}{l}0 \\
5 \\
5\end{array}$ & - & ${ }_{0}$ & $\begin{array}{l} \\
- \\
0\end{array}$ & ${ }_{0}$ & $\begin{array}{l}0 \\
- \\
0\end{array}$ & - & 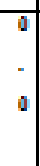 & $\begin{array}{l}- \\
0\end{array}$ & - & $=$ & 0 & 5 & $\begin{array}{l}0 \\
0 \\
0\end{array}$ & $\begin{array}{l}0 \\
5 \\
5 \\
5\end{array}$ & $\begin{array}{l} \\
- \\
0\end{array}$ & $\begin{array}{l}0 \\
- \\
5 \\
5\end{array}$ & $\begin{array}{l}0 \\
- \\
0\end{array}$ & $\begin{array}{l}0 \\
5 \\
5 \\
5\end{array}$ & $\begin{array}{l} \\
- \\
0\end{array}$ & - & \begin{tabular}{|l|}
0 \\
- \\
5 \\
5
\end{tabular} & \begin{tabular}{|l|}
0 \\
- \\
5 \\
5
\end{tabular} & \begin{tabular}{l|}
0 \\
- \\
0
\end{tabular} & $\begin{array}{l}0 \\
0 \\
0\end{array}$ & $\begin{array}{l}0 \\
0\end{array}$ & $\begin{array}{l}0 \\
- \\
0\end{array}$ & $\begin{array}{l}0 \\
- \\
0\end{array}$ & - & $\begin{array}{l}0 \\
- \\
0\end{array}$ & $\begin{array}{l}0 \\
- \\
0\end{array}$ & & & & & 5 \\
\hline 7 & $\begin{array}{l}0 . \\
5 \\
5\end{array}$ & $\begin{array}{l}0 \\
- \\
5\end{array}$ & - & - & $\begin{array}{l} \\
- \\
0\end{array}$ & - & 0 & 0 & 0 & \begin{tabular}{|c|}
1 \\
\end{tabular} & 0 & 0 & 0 & 0 & $\begin{array}{l}0 \\
- \\
0\end{array}$ & $\begin{array}{l}0 \\
0 \\
0\end{array}$ & $\begin{array}{l} \\
- \\
0\end{array}$ & $\begin{array}{l}5 \\
5\end{array}$ & $\begin{array}{l}0 \\
- \\
0\end{array}$ & \begin{tabular}{|l|}
0 \\
- \\
0 \\
0
\end{tabular} & $\begin{array}{l}0 \\
- \\
0\end{array}$ & 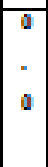 & $\begin{array}{l}0 \\
- \\
5 \\
5\end{array}$ & \begin{tabular}{|l|}
0 \\
- \\
5 \\
5
\end{tabular} &. & $\begin{array}{l}0 \\
0\end{array}$ & \begin{tabular}{|l} 
\\
- \\
0
\end{tabular} & $\begin{array}{l}0 \\
0 \\
0\end{array}$ & 0 & : & $\begin{array}{l}0 \\
- \\
0\end{array}$ & $\begin{array}{l}0 \\
- \\
0\end{array}$ & & & $\mathrm{M}$ & & 5 \\
\hline 8 & $\begin{array}{l}0 . \\
5 \\
5\end{array}$ & $\begin{array}{l}0 \\
5 \\
5 \\
5\end{array}$ & ${ }_{0}^{-}$ & - & $\begin{array}{l} \\
\\
0\end{array}$ & $\begin{array}{l}0 \\
- \\
0\end{array}$ & $\begin{array}{l}0 \\
- \\
0\end{array}$ & 0 & $\begin{array}{l} \\
- \\
0\end{array}$ & $\begin{array}{l}- \\
0\end{array}$ & $\begin{array}{l}0 \\
- \\
0\end{array}$ & 0 & $\begin{array}{l} \\
- \\
0\end{array}$ & $\begin{array}{l}5 \\
5\end{array}$ & $\begin{array}{l}0 \\
0 \\
0\end{array}$ & $\begin{array}{l}0 \\
5 \\
5 \\
5\end{array}$ & $\begin{array}{l}0 \\
0 \\
0\end{array}$ & $\begin{array}{l}0 \\
- \\
5 \\
5\end{array}$ & \begin{tabular}{|l}
0 \\
- \\
0
\end{tabular} & $\begin{array}{l}0 \\
- \\
5 \\
5\end{array}$ & $\begin{array}{l}0 \\
- \\
0\end{array}$ & \begin{tabular}{|l|} 
\\
- \\
0
\end{tabular} & $\begin{array}{l}0 \\
- \\
5 \\
5\end{array}$ & \begin{tabular}{|l|}
0 \\
- \\
5 \\
5
\end{tabular} & $\begin{array}{l}0 \\
- \\
0\end{array}$ & - & $\begin{array}{l}0 \\
0\end{array}$ & $\begin{array}{l}0 \\
0 \\
0\end{array}$ & $\begin{array}{l}0 \\
- \\
0\end{array}$ & $\begin{array}{l} \\
\end{array}$ & $\begin{array}{l}0 \\
0 \\
0\end{array}$ & $\begin{array}{l}0 \\
- \\
0\end{array}$ & $\begin{array}{l}0 \\
0\end{array}$ & & $\overline{\mathrm{M}}$ & & \\
\hline 9 & $\begin{array}{l}0 . \\
5 \\
5\end{array}$ & $\begin{array}{l}0 \\
5 \\
5 \\
5\end{array}$ & - & 0 & $\begin{array}{l} \\
- \\
0\end{array}$ & $\begin{array}{l}0 \\
- \\
0\end{array}$ & $\begin{array}{l}0 \\
- \\
0\end{array}$ & 0 & $\begin{array}{l}0 \\
- \\
0\end{array}$ & : & - & 0 & 0 & 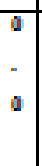 & $\begin{array}{l}0 \\
0 \\
0\end{array}$ & $\begin{array}{l}0 \\
5 \\
5 \\
5\end{array}$ & $\begin{array}{l}0 \\
- \\
0\end{array}$ & $\begin{array}{l}5 \\
5 \\
5\end{array}$ & \begin{tabular}{|l}
0 \\
- \\
0
\end{tabular} & \begin{tabular}{|l|}
0 \\
5 \\
5 \\
5
\end{tabular} & $\begin{array}{l}0 \\
0 \\
0\end{array}$ & - & \begin{tabular}{|l|}
0 \\
- \\
5 \\
5
\end{tabular} & $\begin{array}{l}0 \\
- \\
5 \\
5\end{array}$ & $\begin{array}{l} \\
- \\
0\end{array}$ & $\begin{array}{l}0 \\
0\end{array}$ & $\begin{array}{l}0 \\
0 \\
0\end{array}$ & $\begin{array}{l}0 \\
0 \\
0\end{array}$ & $\begin{array}{l}0 \\
- \\
0\end{array}$ & 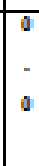 & $\begin{array}{l}0 \\
- \\
0\end{array}$ & 0 & - & & & & \\
\hline$\overline{4}$ & $\begin{array}{l}0 . \\
5 \\
5\end{array}$ & 5 & - & - &. & - & 0 & 0 & 0 & 0 & - & 0 & 0 & 5 & $\begin{array}{l}0 \\
0 \\
0\end{array}$ & $\begin{array}{l}0 \\
5 \\
5 \\
5\end{array}$ & $\begin{array}{l}0 \\
- \\
0\end{array}$ & 0 & \begin{tabular}{|l}
0 \\
- \\
0
\end{tabular} & $\begin{array}{l}0 \\
5 \\
5 \\
5\end{array}$ & $\begin{array}{l}0 \\
0 \\
0\end{array}$ & - & $\begin{array}{l}0 \\
\\
0\end{array}$ & $\begin{array}{l}0 \\
5 \\
5 \\
5\end{array}$ & -1 & - & $\begin{array}{l}0 \\
0 \\
0\end{array}$ & $\begin{array}{l}0 \\
0 \\
0\end{array}$ & 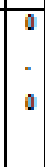 & 0 & $\begin{array}{l}0 \\
- \\
0\end{array}$ & - & & & $\mathrm{M}$ & $x$ & \\
\hline 4 & $\begin{array}{l}0 . \\
5 \\
5\end{array}$ & 5 & - & - & 0 & - & 0 & 10 & 0 & 0 & - & 0 & 0 & 5 & $\begin{array}{l}0 \\
0 \\
0\end{array}$ & $\begin{array}{l}0 \\
0 \\
0\end{array}$ & $\begin{array}{l}0 \\
- \\
0\end{array}$ & 5 & $\begin{array}{l}0 \\
- \\
0\end{array}$ & $\begin{array}{l}0 \\
- \\
0\end{array}$ & $\begin{array}{l}0 \\
0 \\
0\end{array}$ & - & \begin{tabular}{|l} 
\\
- \\
5 \\
5
\end{tabular} & $\begin{array}{l}0 \\
- \\
0\end{array}$ & $\begin{array}{l}0 \\
- \\
0\end{array}$ & $\begin{array}{l}0 \\
- \\
0\end{array}$ & - & - & 0 & ; & $\begin{array}{l}0 \\
- \\
0\end{array}$ & o & - & & & & \\
\hline
\end{tabular}




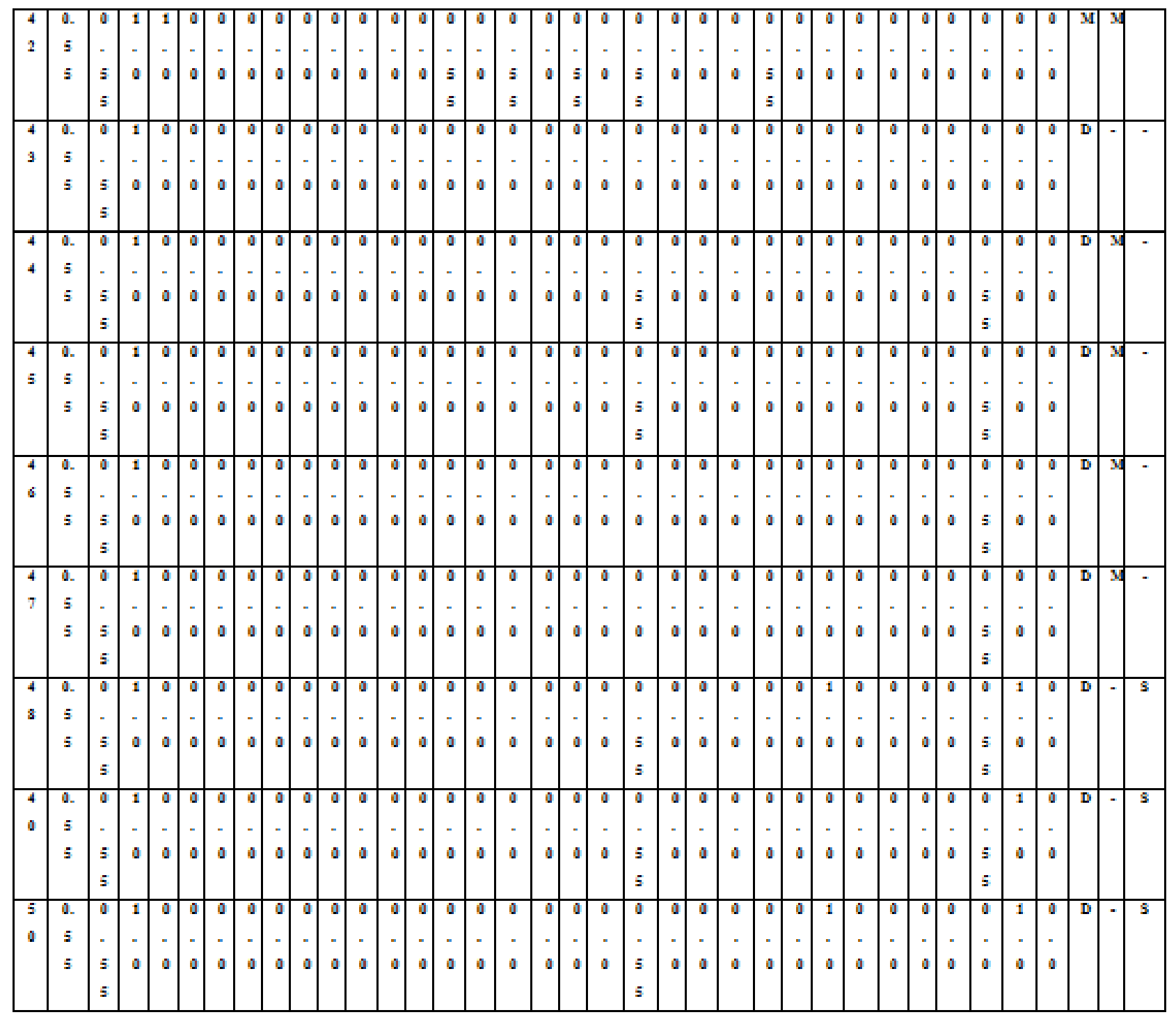

\section{A. General Symptoms Confrimation Of Fever Type}

Applying eq(3) to the general symptoms entries(first five rows) in table4 above, the results is presented graphically in fig4 below. We can clearly see the demarcation between the various types of fever confirmed (malaria, dengue, typhoid, lass and yellow fever), as each patients in each group of fever type have same general symptoms value. Also, a group with higher general symptom value includes all symptoms of other fever types with lower general symptom value.. For example, the symptoms difference between 1 to $\mathrm{p} 10$, and $\mathrm{p} 11$ to $\mathrm{p} 19$ are symptoms not reported by patients $\mathrm{p} 1$ to $\mathrm{p} 10$ but are reported by $\mathrm{p} 11$ to $\mathrm{p} 19$ patients who also reported symptoms of $\mathrm{p} 1$ to $\mathrm{p} 10$ as well. Similarly, $\mathrm{p} 1$ to p10 patients has reported all symptoms reported by p20 to p30 patients and some extra symptoms not reported by p20 to p30 patients. In addition, p31 to p42 patients has reported more symptoms than $\mathrm{p} 43$ to $\mathrm{p} 50$ patients but $\mathrm{p} 31$ to $\mathrm{p} 42$ patients also reported all symptoms reported by p43 to p50 patients as well. Furthermore, p1 to p10 patients has reported slightly few symptoms than p43 to p50 patients, although p43 to p50 patients all reported symptoms reported by $\mathrm{p} 1$ to $\mathrm{p} 10$ patients as well. Hence, increasing the accuracy of fever type diagnosis with physiological symptoms. 
fever types confirmation results

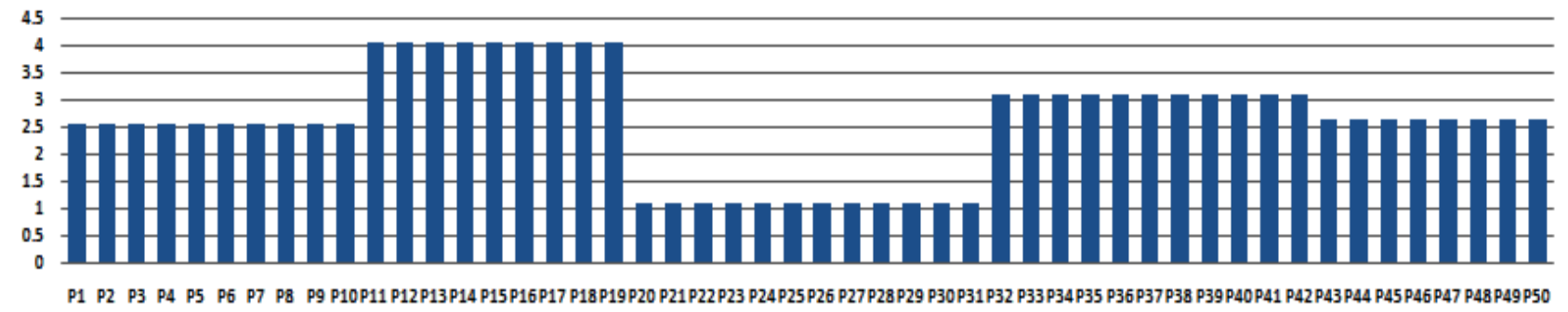

-GENERAL SMMPTOM SUMMATIONS

Fig.4. General symptoms summation and the type of fever confirmed

Furthermore, an extension of the general symptom analysis, applying eq(3) is presented in fig4 below, showing all the confirmed types of fever. Here, dengue and yellow fever infected patients is nine (9) each, malaria, and typhoid infected patients is 11 patients each, and Lassa fever infected patients is 10 .

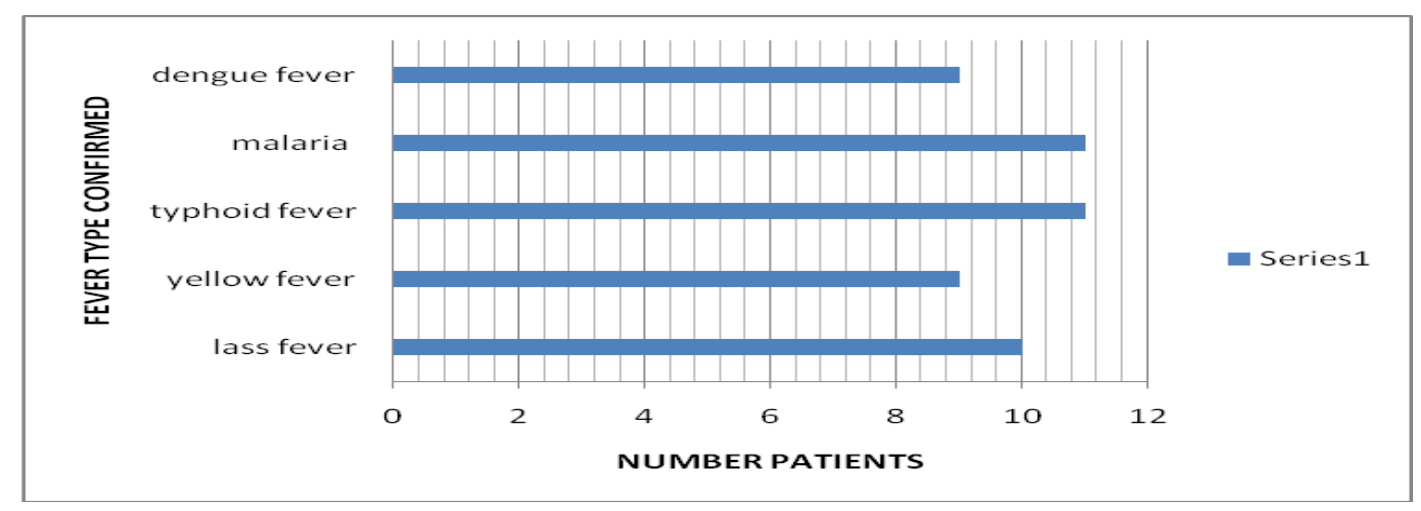

Fig.5. Type of fever confirmed with number of patients.

\section{B. Unique Symptoms Confirmation Of Level Of Infection}

Furthermore, an analysis on the confirmed type of fever to determine their level of infection using unique symptoms reported by patients and applying eq(4). The summary of all unique symptoms reported by all fifty patients are analyzed in the bar-chart FIG6 below, to better visualize the unique symptoms patterns. The graph clearly pictures a vast variation in the unique symptoms reported by all patients irrespective of the type of fever confirmed. For example patients p1, p19, p20, and p43 did not report any other symptoms, irrespective of the type fever confirmed Hence further supporting the variation of level of infection among patients of the same type of fever and different type types of fever as either severe or mild.

Applyign eq(3) to table4 above, for each patient, the summation of general symptom reported is obtained and presented graphically in fig4. Clealry we can see that each group of patients has equal number of general symptom reported value, that distinugish each of the types of fever under study. Furthermore, the type of fever confirmed for each group in fig4 is presented in fig5 . Therefore, confirming malaria and typhoid fever being the highest with 11 patient ptients each. While lassa fever had 10 patients and the The laeast was dengue and yelow fever with 9 patients each.

Furthermore, Applying eq(4) to table4 above, the total unique symptoms values of unique symptoms reported by each patient after corresponding to the type of fever confirmed above is obtained and graphically presented in fig6. It can be observed that, though at fever type confirmation stage in fig $4 \& 5$ above, all patients has the same general symptom total value for each types of fever confirmed clearly dinstinuishing the various types of fever, but the reverse is the case in confirming the level of infection. Clearly we observe visually the unequal total unique symptom values in fig6, which is an evidence to the variation to the level of infection of the confirmed type of fever for each patient, further supported by the variation in the number of unique symptoms reported by each patients.

Furthermore applying eq(2) to table4 is a combination of the result from applying eq(3) and eq(4) to table 4 above, which is also equivalent to the combination of fig 4 and fig6. This outcome is the total integrated diagnosis result for each patient with confirmed type of fever infection and level of infection, which is represented in fig7. We can clearly observe great variation between patient symptoms values infig6 and fig7, which further implies that the level of infection has to do with the number of severe and mild symptoms irrespective to general or unique symptom classification since this only helps in stepwise diagnosis. A patient may have higher total symptom value but will be in mild state because he/she has not reported occurrence of any additional 
severe symptoms beside the fever type confirmation general symptoms. Hence we can firmly confirm with fig6 , the level of infection varies for all patients through their unique symptom and not the general symptoms that comfirms type of fever they are suffering from. We can also affirm the difficulty in using physiological symptoms for fever diagnosis without considering the impact of symptom relationship as a confirmation mechanism has significan impact on diagnosis result accuracy based on the variation in fig7.

Unique symptom infection level of confirmed type of fever

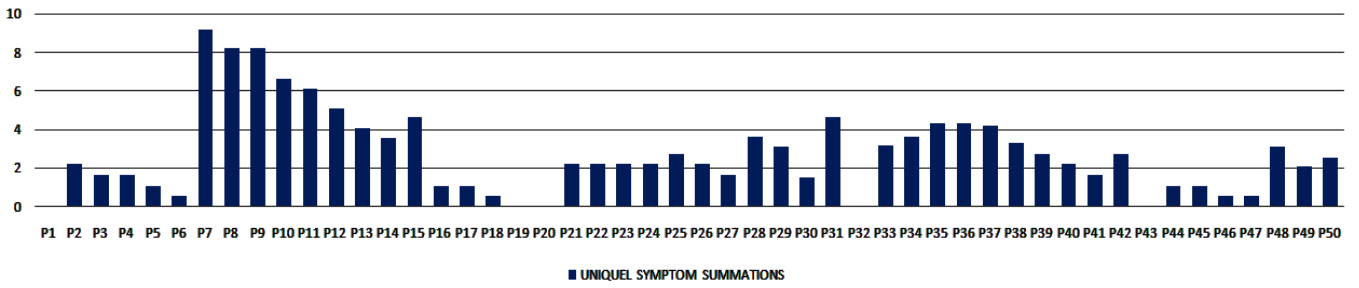

Fig.6. Unique symptoms reported among fever patients

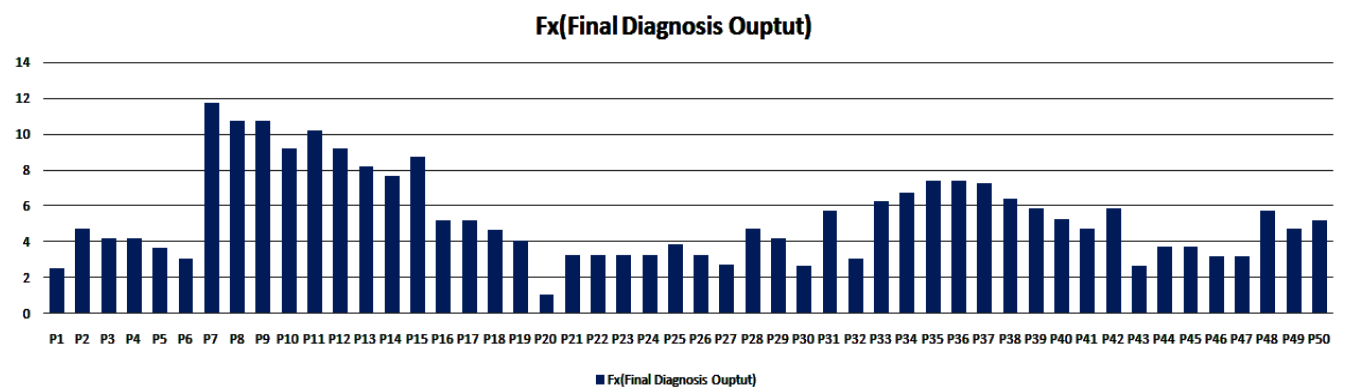

Fig.7. Finally diagnosis output with total symptoms values for all patients confirming the type of fever and the level of infection

Further analysis on the results from implementing the e-fever portal based on the fuzzy-based multi-fever symptoms classifier, is presented in fig8. There are 5, 4, and 1 patient having lass fever infected at mild, severe and unknown level respective. In addition, there are 4,4, and 1 patients having yellow fever infected at mild, severe and unknown level respectively. In addition, there are 7,4 , and 1 patients having typhoid fever infected at mild, severe and unknown level respectively. Furthermore, there are 5, 5, and 1 patient having malaria fever infected at mild, severe and unknown level respectively. In addition, there are 4,3 , and 1 patient having dengue fever infected at mild, severe and unknown level respectively.

\section{CONFIRMED FEVER LEVEL OF INFECTION ESULT}

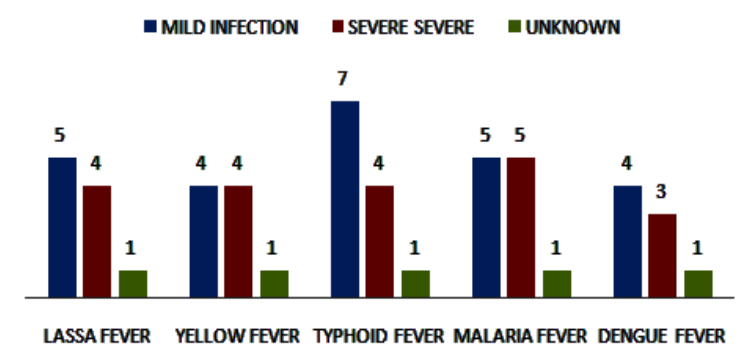

Fig.8. Summary analysis of e-fever portal diagnosis results
Furthermore, analysis on Fig8 above, the number of mild fever patients as presented graphically in fig9a. Clearly we can infer that $16 \%, 20 \%, 16 \% 28 \%$, and $20 \%$ of all 50 fever infected patients are mildly infected with dengue, Lassa, yellow, typhoid and malaria fever. Which if early therapy is administered can save them from entering into severe state. The number of severely infected fever patients is analyzed I fig9b , which clearly indicates, $15 \%, 20 \%, 20 \%, 20 \%$, and $25 \%$ of all fifty fever infected patients are mildly infected with dengue, Lassa, yellow, typhoid and malaria fever. There are also some cases of unknown level of fever infection, although with confirmed type of fever infection. These are indicated with "--" in table4 and are further analyzed in fig9c. There is $20 \%$ of each group of patients in the five types of fever confirmed without any level of infections.

\section{MILD INFECTION}

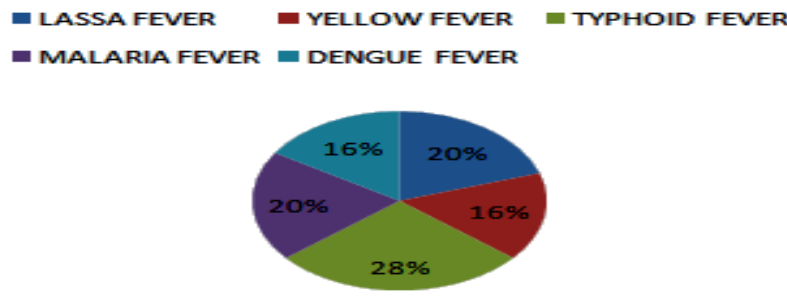

Fig.9a. Mild level fever infected patient's analysis 


\section{SEVERE INFECTION}

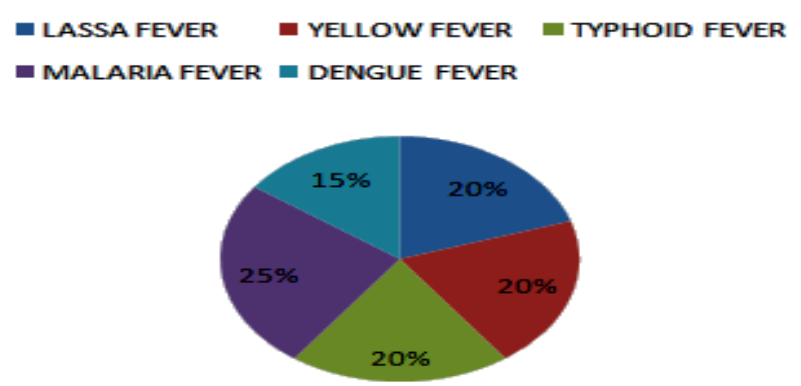

Fig.9b. Severe level fever infected patient's analysis

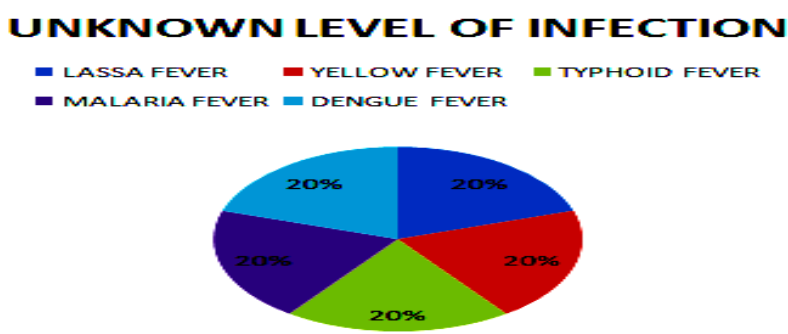

Fig.9c. Unknown level fever infected patient's analysis

\section{System Acceptability}

The system acceptability test was conducted with the same 50 patient administered a structured questionnaire comprising of five direct questions, to ascertain the system acceptability and usability of the system. In this section, 50 responses was collected, of which 44 was valid and 6 was invalid, due to multiple response selected, which cannot be accepted. From the fig10a, we can infer that $60 \%$ strongly agreed that the system is quit easy to use, while $28 \%$ agreed also that it is easy to use. The other $12 \%$ represents the invalid unrepresented population responses. In addition, the cost effectiveness of the system was measured from user's perspective in relation to visiting a medical expert for diagnosis, as depicted in Fig10b. During which 50 questionnaires were administered, out which 3 were invalid, 47 were valid, out of which $80 \%$ responded strongly agreed to the cost effectiveness of the e-fever portal compared to visiting a medical. While $14 \%$ agreed and the remaining $6 \%$ are the invalid population due to multiple responses. Furthermore, the accessibility of e-fever portal for diagnosis of fever was compared with medical expert, through the administered questionnaire, considering a population of 50 respondents, as presented in fig10c. During which, $78 \%$ strongly agreed to the accessibility of e-fever portal than medical expert, citing booking and appointment and visiting a medical expert a major bottleneck on the accessibility of medical expert. While internet availability and a mobile technology as a major factor enhancing the accessibility of e-fever portal. Also, $10 \%$ agreed stating same reasons with the $78 \%$ strongly agreed. In addition, $8 \%$ responded indifference. While the remaining $4 \%$ disagreed, mostly because they lived in the city where all medical facilities are always available as their reason.

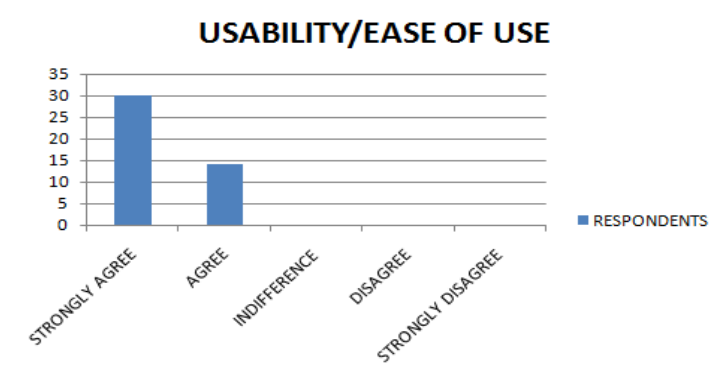

Fig.10a. Usability analysis

COST EFFECTIVENESS

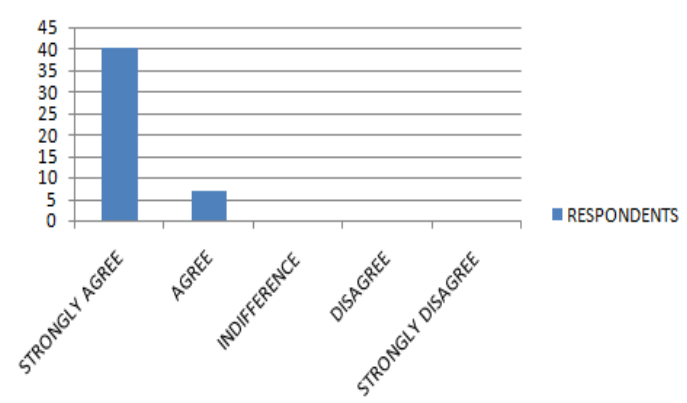

Fig.10b. Cost effectiveness analysis

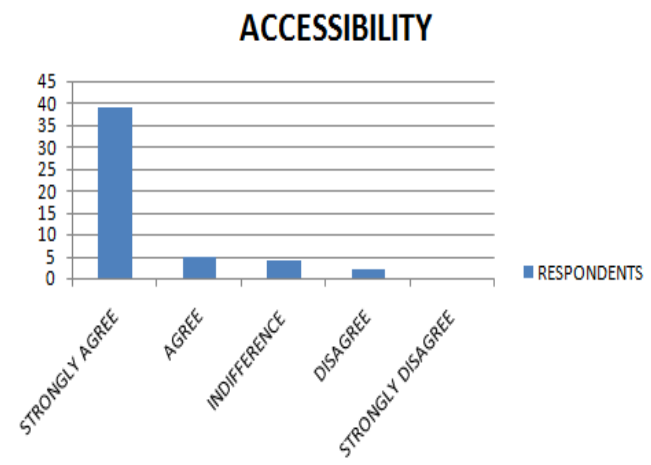

Fig.10c. Accessibility analysis

\section{CONCLUSSION}

In conclusion, an integrated expert fever diagnosis system implementing a novel fuzzy-based multi-fever symptom classifier has been developed tested and evaluated for acceptability by users. A comparative analysis study on existing fever diagnosis systems was carried out and the result clearly showed that the e-fever portal is quiet novel with multi-fever symptoms relationship capability integrated for accurate diagnosis. In addition the test analysis results clearly shows that irrespective of symptom relationship between the various types of fever (dengue, malaria, typhoid, Lassa, and yellow fever), the fuzzy rule accurately determines the type of fever first, then ascertain the level of infection as either mild or severe. Furthermore, the acceptability of the e-fever portal was evaluated using structured questionnaires, administered to the 50 patients. The 
responses clearly indicate high ease of usage, citing user interface friendliness and simple look and feel of the system interface. Also, respondent highly consented to fast accessibility to e-fever portal than medical expert, strongly agreeing and citing the availability of mobile technology and internet connectivity. Furthermore, respondents strongly agreed and agreed more to the cost effectiveness of e-fever portal compared to medical expert citing the exemption of transportation and other logistics involved in visiting a medical expert as an advantage of e-fever portal, in addition to the extra cost of consultation and unnecessary wet lab diagnosis in some cases. Hence the e-fever portal promises to improve fever diagnosis results, as a computer aided expert medical diagnosis system, considering symptom interlink among various fever types. In addition to improve accessibility of fever diagnosis service through web delivery to all, location independent globally.

\section{REFFERENCES}

[1] James M Heilman, Jacob De Wolff, Graham M Beards, Brian J Basden, Dengue fever: a Wikipedia clinical review, Open Medicine; vol8 issue4 page105, 2014.

[2] ichirokuran, dengue hemorrhagic fever with special emphasis on immunopathogenesis, journal of comaparative immunology and microbiologyand infectious disease, 2007, doi/10.1016/j.cimid.2007.05.010

[3] RiazA,AmirRiazDengue fever: Prevention most recommended, theHealth Vol 2, Issue 2, pp33-34, 2011

[4] Fever: Its Biology, Evolution, and Function. Princeton University Press.. p. 57. ISBN 9781400869831. 2015

[5] Garmel, Fever in adultsGus M, An introduction to clinical emergency medicine (2nd ed.). Cambridge: Cambridge University Press. p. 375. ISBN 0521747767.

[6] Section on Clinical Pharmacology and, Therapeutics; Committee on, Drugs; Sullivan, JE; Farrar, HC "Fever and antipyretic use in children.".Pediatrics127 (3): 580-7. 2011.

[7] Garmel, edited by S.V. Mahadevan, Gus M. An introduction to clinical emergency medicine (2nd ed.). Cambridge: Cambridge University Press. p. 5. 2012.

[8] Richardson, M; Purssell, E "Who's afraid of fever?". Archives of Disease in Childhood 100 (9): 818-20. September 2015.

[9] Garmel, edited by S.V. Mahadevan, Gus M. An introduction to clinical emergency medicine (2nd ed.). Cambridge: Cambridge University Press. p. 401. 2012.

[10] Kiekkas, P; Aretha, D; Bakalis, N; Karpouhtsi, I; Marneras, C; Baltopoulos, GI "Fever effects and treatment in critical care: literature review.". Australian Critical Care 26 (3): 130-5. August 2013.

[11] Niven, Daniel J.; Gaudet, Jonathan E.; Laupland, Kevin B.; Mrklas, Kelly J.; Roberts, Derek J.; Stelfox, Henry Thomas "Accuracy of Peripheral Thermometers for Estimating Temperature". Annals of Internal Medicine 163 (10): 768.17 November 2015.

[12] Barone JE "Fever: Fact and fiction". J Trauma Vol. 67 Issue2, PP406-409, (August 2009)..

[13] jyh-sing roger jang, chuen-tsai.sun, and eijl mizutani, Neurofuzzy and soft computing: A computational approach to learning and machine intelligence, Eastern Economy Edition, 17 November 2015.

[14] Oguntimilehin A, Adetunmbi A.O. and Olatunji K.A., A Machine Learning Based Clinical Decision Support
System for Diagnosis and Treatment of Typhoid Fever, International Journal of advanced Research in Computer Science and Software Engineering Volume 4, Issue 6, pp 961 969, June 2014.

[15] Tarig Faisal, Mohd Nasir Taib, Fatimah Ibrahim, Adaptive Neuro-Fuzzy Inference System for diagnosis risk in dengue patients (PDF) Accessed 21/03/2016

[16] Sharifah Hanis, and BT Syed Ahmad, an expert system to track dengue fever A thesis submitted in fulfillment of the requirement for the award of the degree of Bachelor of Computer Science (PDF) Accessed 21/03/2016

[17] O.W. Samuel, M.O. Omisore, B.A. Ojokoh, A web based decision support system driven by fuzzy logic for the diagnosis of typhoid fever, Expert Systems with Applications Journal, Expert Systems with Applications Vol.40, PP 4164-4171, 2013.

[18] X.Y. Djam1, G. M. Wajiga, Y. H. Kimbi and N.V. Blamah,A Fuzzy Expert System for the Management of Malaria, International Journal of Pure and Applied Sciences and Technology, Vol.5, Issue2, pp84-108, 2011.

[19] Priynka Sharma, DBV Singh, Manoj Kumar Bandil and Nidhi Mishra, Decision Support System for Malaria and Dengue Disease Diagnosis (DSSMD), International Journal of Information and Computation Technology.Volume 3, Number 7, pp. 633-640, 2013.

[20] Adetunmbi A.O, Oguntimilehin A, Falaki S.O., webbased medical assistant system for malaria diagnosis and therapy GESJ: Computer Science and Telecommunications, Vol. 1 No.33, 2012, PP42-53, June 2014

[21] sunday tunmibi, oriyomi adeniji, ayooluwa aregbesola, and ayodeji dasylva, a rule based expert system for diagnosis of fever, International Journal of Advanced Research, Volume 1, Issue 7, 2013,pp343

[22] Jimoh R.G., Awotunde J.B., Babatunde A. O., Ameen, A. O \& Fatia O.W, Simulation Of Medical Diagnosis System For Malaria Using Fuzzy Logic , Computing, Information Systems, Development Informatics \& Allied Research Journal Vol. 5 No. 2., Pp59-68, June 2014

[23] Ojeme Blessing Onuwa, Fuzzy Expert System For Malaria Diagnosis, Oriental Journal Of Computer Science \& Technology, Vol. 7, No. (2), , pp 273-284, June 2014

[24] Normile D. Surprising new dengue virus throws a spanner in disease control efforts. Tropical medicine Science, Vol342, issue 6157, page415, 2013

[25] Sue E. Huether, Pathophysiology: The Biologic Basis for Disease in Adults and Children (7 ed.). Elsevier Health Sciences. p. 498, 2014..

[26] Kalyani Baghel, Neeraj Mehta, A Web Based Fuzzy Expert System for Human Disease Diagnosis, International Journal Of Engineering And Computer Science Volume 4 Issue 9, Pp. 14248-14253, Sep 2015

[27] Nidhi mishra, and P. Jha, A review on the applications of fuzzy expert system for disease diagnosis, international journal of advanced research in engineering and applied sciences vol. 3, no. 12 pp 28 -43, December 2014.

[28] Asogbon MG, Samuel OW, Omisore MO and Awonusi O, Enhanced Neuro-Fuzzy System Based on Genetic Algorithm for Medical Diagnosis, Journal of Medical Diagnostic Method Volume 5, Issue 1-1000205. doi: 10.4172/2168-9784.1000205

[29] S. Govinda Rao M. Eswara Rao D. Siva Prasad Diagnosis Rule-Based Expert Systems International Journal of Engineering Research \& Technology (IJERT) Vol. 2 Issue 8, pp551-561, August - 2013 


\section{Authors' Profiles}

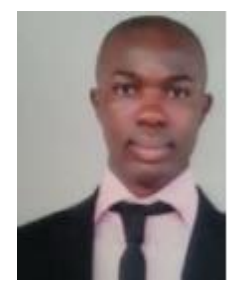

Ighoyota Ben. Ajenaghughrure, recently received his masters in computer science degree from Vels University India, in 2016 may. While he obtained his bachelor's in computer science from Delta State University. His research interest are in artificial intelligence, Human computer Interaction, health and business information systems, Trust, Social and security computing, and computer vision application. $\mathrm{He}$ is currently the head of information technology department of Wetland Microfinance Bank Nigeria Ltd,

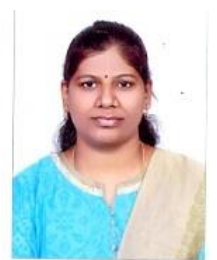

Dr. P. Sujatha is working as Head and Associate Professor in the Department of Computer Science, School of Computing Sciences, Vels University, Chennai, Tamil Nadu, India which is well known University. She has 17 years of teaching experience in both UG and PG Level. She has received her M.Sc (Computer Science) degree from Madurai Kamaraj University, M.Phil degree from Periyar University, Tamil Nadu, India and Ph.D degree in Computer Science from Vels University, Chennai, Tamil Nadu, India. Her research interests include Image Processing, Network Security and Data Mining. She has produced 6 M.Phil Scholars. Currently she is guiding $8 \mathrm{Ph} . \mathrm{D}$., Research Scholars and 3 M.Phil Research Scholars in Vels University.

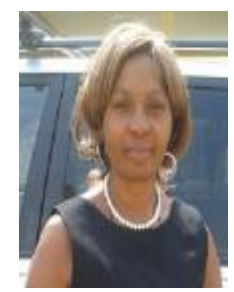

Dr. Maureen I. akazue is a Lecturer in the Department of Mathematics \& Computer Science, Delta State University, Abraka, Delta State, Nigeria. She received Master of Information Science degree in 2001 from University of Ibadan, Oyo State, Nigeria, M.Sc. Computer Science in 2008 and $\mathrm{PhD}$ Computer Science in 2014, both from University of Benin, Edo State, Nigeria. Her research interests are HCI, Enhancing Customer's satisfaction, Online fraud prevention, Security challenges and Computer Solutions, business information systems, and Trust computing.

How to cite this paper: Ighoyota Ben Ajenaghughrure, P. Sujatha, Maureen I. Akazue, "Fuzzy Based Multi-Fever Symptom Classifier Diagnosis Model", International Journal of Information Technology and Computer Science(IJITCS), Vol.9, No.10, pp.13-28, 2017. DOI: 10.5815/ijitcs.2017.10.02 